\title{
Schrift an Bild im World Wide Web \\ Articulirte Pixel und die schweifende Unbestimmtheit des Vorstellens
}

\begin{abstract}
Wie wirkt sich die je spezifische Materialität von Sprache auf die Gestalt unserer Zeichen und damit auf die Bildung unserer Vorstellungen aus? Das wird hier am Beispiel von Schrift im World Wide Web untersucht. Unter dem Einfluss technisierter Materialität, Medialität und Multimodalität bildet sich eine Schriftlichkeit aus, deren Erscheinungsform und Bedeutung aus dem semiotischen Zusammenspiel von Sehflächen-Design, Bild und Text erwächst. Vor dem Hintergrund einer medial gestützten Geschichte kommunikativer Abstraktion, in deren Verlauf Leib und Seele im Bewusstsein auseinander traten, und anhand charakteristischer Beispiele aus dem WWW werden Entstehungsbedingungen, Eigenschaften und Leistungen solch ,tertiärer Schriftlichkeit' erläutert.
\end{abstract}

\section{Semiotechnik - what else?}

Bitte schauen Sie zunächst den Werbefilm „Mistaken“ im World Wide Web an: www.nespresso-whatelse.com. ${ }^{1}$ Was haben Sie hier gesehen?

\subsection{Erstes Beispiel: Situiert und verkörpert}

Wir haben eine künstlich arrangierte Situation sozialer Interaktion erlebt, die in jeder Hinsicht möglichst alltäglich erscheinen soll (und in die wir durch die Hintergrundgeräusche als voyeuristisch teilnehmende Beobachter möglichst authentisch einbezogen werden sollen), obgleich wir natürlich wissen, dass es um Werbung geht, die sich versteckt: Der berühmte Schauspieler und Regisseur George Clooney inszeniert sich als zuerst richtig, dann vermeintlich irrtümlich erkannte Alltagsperson. Augenzwinkernd spielt das filmische Theater im Theater mit der fragilen Balance von Fiktion und Wirklichkeit, Maskerade ohne Maske.

Medial wird hier natürliche Face-to-face-Kommunikation ${ }^{2}$ vorgeführt mit allen deren Kennzeichen: Personen in ihrer gesamten Körperlichkeit interagieren in einer ganz bestimmten (typischerweise einzigartigen, unwiederholbaren) Situation. In der englischsprachigen Literatur gibt es dafür die knappen Schlagwörter embodiment and situatedness (Verkörperung und Situiertheit). Beide gelten der modernen Cognitive Science (und in deren Rahmen

\footnotetext{
$\overline{1}$ Er stammt aus dem Jahre 2008 und findet sich als 31-Sekunden-Film mehrfach auch in www. youtube.com, z.B. über die Suchwörter „Nespresso Clooney 2008“.

2 Weinrich (1988, S. 81) nennt das „Blickstellung“.
} 
auch der kognitiven Linguistik) als die charakteristischen Merkmale ,natürlicher ${ }^{6}$ Kommunikation. ${ }^{3}$ Auf den ersten Blick erscheinen diese beiden Merkmale im Beispiel auch so. Auf den zweiten Blick (den Blick hinter die Kulissen) wissen wir natürlich, dass gerade diese beiden Eigenschaften hier als scheinbar authentisch nur simuliert wurden (denn mediale Kommunikation ersetzt ja gerade ,natürliche' Unmittelbarkeit). Auf den dritten Blick jedoch können wir in unserem eigenen Leben beobachten, wie mediale Kommunikation immer tiefer in unseren Alltag einwandert und ,natürliche' Face-toface-Kommunikation durchdringt und umgestaltet.

Je weiter menschliche Geschichte voranschreitet, desto mehr wird Kommunikation (wie die meisten anderen Lebensbereiche auch) technisiert und dadurch verändert.

\subsection{Zweites Beispiel: Technische Simulation und Schrift}

Die folgenden Überlegungen gelten einem ganz kleinen Aspekt innerhalb dieses Jahrtausende alten und sich immer schneller beschleunigenden Vorgangs zunehmender Technisierung von Kommunikation und seiner Auswirkungen auf Sprache, nämlich der Veränderung von Schriftlichkeit auf hochauflösenden Bildschirmen. Es geht also nicht um die äußere Erscheinung von Schrift, ${ }^{4}$ sondern um Schriftlichkeit (im Gegensatz etwa zu Mündlichkeit und Bildlichkeit) als allgemeine Ausdrucksform. Ich möchte zeigen, wie Schriftlichkeit sich ändert unter dem Einfluss dreier zunehmend technisierter Faktoren, nämlich MATERIALITÄT, MEDIALITÄT UND MULTIMODALITÄT.

Betrachten Sie dieses Beispiel aus der gleichen Werbekampagne:

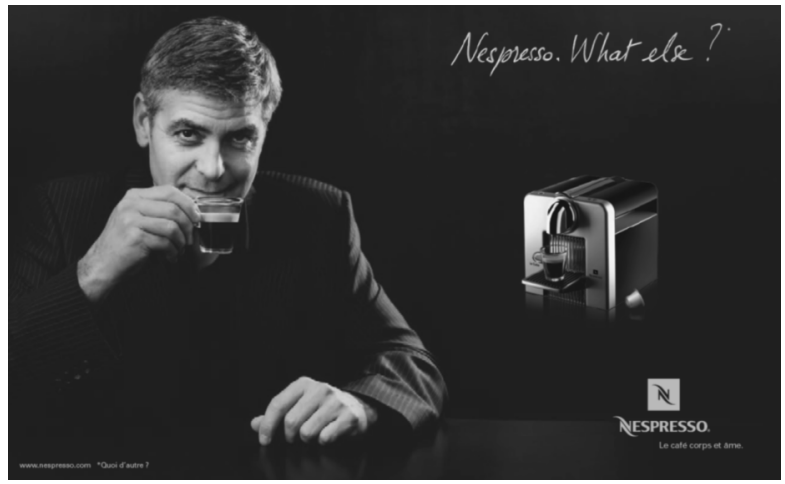

Abb. 1: Nespresso-Werbung (Werbeplakat am Flughafen Paris-Orly Dezember 2008) ${ }^{5}$

Vgl. z.B. Gibbs (2006).

Für Schriftschnitt, Zeichensatz, Typographie, Kalligraphie etc. eröffnen sich durch Digitalisierung auch neue Perspektiven, doch das ist ein Thema für sich. Vgl. z.B. Spitzmüller (2006) und Wehde (2000).

5 www.effie.fr/palmares/2007/nespresso/Affiche/nespresso_affiche2.pdf (Stand: Januar 2009). 
Unsere schriftgewohnte Leseblickrichtung von links nach rechts verknüpft das Glas Kaffee an Clooneys Lippen (body) mit dem perspektivisch kleineren, sonst gleichen Glas an der Kaffeemaschine (Technik). In diese elliptisch konzentrierte, minimalistische Situation werden wir durch Clooneys freundlichen Blick einbezogen. Unmittelbar nach diesem Bildfang nehmen wir rechts oben ein handschriftliches Textfeld wahr. Mit Clooney und Kaffeemaschine verbindet es sich zu einem imaginären Dreieck. So wirkt es als Sprechblase, das mit seinem elliptischen Slogan-Text „Nespresso. What else?“ die medial fehlende Mündlichkeit ersetzt. Clooney scheint uns direkt anzusprechen. Sein Ausdruck (vornehmlich mimisch übers Gesicht), die Darstellung (vornehmlich über das Bild der Kaffeemaschine) und der Appell (vornehmlich über den Wortlaut) sind fein säuberlich auf die drei Ecken verteilt, als sollte Bühlers (1934, S. 28) Organon-Modell illustriert werden. In diesem wohlgeplanten Zusammenspiel trägt Schrift keine Bedeutung für sich allein, sondern nur in Verbindung mit den Bildern, hier also aus der simulierten Interaktionssituation heraus. Weil wir das meiste aus der Situation verstehen, kann der Text so knapp und grammatikarm sein: drei lexikalische Morpheme ohne grammatische Markierung und zwei Interpunktionszeichen. MATERIELLE, MEDIALE und MODALE Konstellationen prägen den Text.

\subsection{Thema und Gegenstand des Aufsatzes}

Ich möchte in diesem Beitrag darlegen, wie sich die materielle Grundlage von Sprache auf die Gestalt unserer Zeichen auswirkt und damit auf die Bildung dessen, was wir ausdrücken, also auf unsere Vorstellungen.

Dabei konzentriere ich mich auf diejenigen Fallgruppen, in denen sichtbare öffentliche Kommunikation sich heute am häufigsten vollzieht, nämlich auf Text-Bild-Verbindungen, wie sie uns auf Sehflächen fast überall umgeben. ${ }^{6}$ Sehflächen sind Flächen, auf denen Texte und Bilder in geplantem Layout gemeinsame Bedeutungseinheiten bilden. ${ }^{7}$ Hier wiederum

Christina Weiss bemerkte schon 1984 zu Beginn des Vorwortes zu ihrer Dissertation von 1982: „Die Orte der Sprache sind allgemeiner geworden. Sprache begegnet uns körperhafter und bildhafter denn je in der unmittelbaren Umwelt. Wir leben inmitten eines riesigen Sprachgewimmels und Wortgedröhns, das wir pausenlos - routiniert - verarbeiten: aus Bildund Tongeräten, aus gedruckter Massenware, von Plakaten und Schildern herab, von einer längst überdosierten Menge der Gegenstandsverpackungen rückt uns die Sprache auf Schritt und Tritt zu Leibe. Die öffentliche Textbegegnung ist sehlastig. Die Sprache, die uns umfängt, nehmen wir in der Mehrzahl als Bild bzw. in der Kombination mit Bildern wahr. Wir sehen die Sprache, bevor wir sie lesen, wir registrieren - bewußt oder unbewußt - diverse Schrifttypen, -größen und -farben. Buchstaben und Wörter fügen sich nahtlos in die Reihe der Alltagsalphabete aus Bildzeichen und Sehsignalen." (Weiss 1984, S. 7).

Siehe Schmitz (im Druck). „Seh-Texte“ hingegen nennt Weiss (1984, S. 16) - im Anschluss an Ferdinand Kriwet (dazu Weingart 2007) - künstlerische Arbeiten, „,welche durch die Reflexion auf die Möglichkeiten ihres Ausdrucksmediums in dessen Grenzbereiche vordringen 
beschränke ich mich auf die avantgardistischste Kommunikationsform, das World Wide Web. Es setzt Trends auch für andere, ältere und trägere Kommunikationsformen (z.B. Plakate, Zeitschriften, Schulbücher u.a.). Und ich kümmere mich weniger um Beziehungen zwischen Text und Bild (die zu untersuchen auch sehr spannend ist) ${ }^{8}$ als vielmehr um Veränderungen an Schriftlichkeit selbst: Wie reagiert Schrift auf die technisierte MATERIALITÄT, MEDIALITÄT UND MULTIMODALITÄT in Hypermedia? Folglich geht es um „Schrift an Bild im World Wide Web“.

Das gezeigte Beispiel (Abb. 1) steht vertretungsweise für viele. Sie alle treten an einer bestimmten Stelle in einer großen historischen Entwicklung auf, die - vordergründig unsichtbar - in sie eingeschrieben ist. Es ist eine medial gestützte Entwicklung kommunikativer Abstraktion, in deren Verlauf Leib und Seele im Bewusstsein auseinandertreten.?

\subsection{Gliederungsausblick}

Auf diese Geschichte gehe ich im 2. Teil kurz ein. Im dann folgenden 3. Teil plädiere ich dafür, beim Nachdenken über Zeichen nicht deren Körper zu vergessen. Teil 4 unterscheidet Material, Medium und Kommunikationsform als Ressourcen und stets wirksame Rahmenbedingungen menschlicher Kommunikation. Teil 5 beschreibt deren ausdrucks- und Teil 6 deren inhaltsbezogene Auswirkungen auf Sehflächen im Web. Der abschließende 7. Teil diskutiert performative Effizienz und Lust unter den Bedingungen technisch erzeugter Situiertheit von Kommunikation.

\section{Kommunikative Abstraktion durch Technik}

\subsection{Unmittelbare Kommunikation}

1) Kommunikation verläuft prototypisch und zuerst interaktiv mit allen Sinnen zwischen zwei oder mehreren Partnern, die sich am selben Ort und zur gleichen Zeit in einer gemeinsamen Situation, also auch in einem gemeinsamen Wahrnehmungsraum befinden. In menschlichen Gesellschaften bildet sich dafür phylo- wie ontogenetisch mündlich-verbaler Austausch als

und in gleicher Weise mit bildnerischen wie mit sprachlichen Mitteln arbeiten“, insbesondere visuelle Poesie. Vgl. auch Linck/Rentsch (Hg.) (2007), daneben Eckkrammer/Held (Hg.) (2006).

8 Allgemein dazu siehe z.B. Stöckl (2004); zu Text-Bild-Beziehungen im Www Runkehl (2005), Schmitz (2003), Siever (2005), Storrer (2004); zu Bild-Diskursen im Netz Meier (2008); vgl. auch Vlasic/Schweiger (1998).

9 Die Entwicklung des abendländischen Dualismus von Leib/Körper vs. Seele/Geist mit seinen zeichentheoretischen Implikationen braucht hier nicht detailliert nachvollzogen zu werden (vgl. dazu - etwa neben Hörisch 1992 - die Artikel „Doppelnatur“, „Leib, Körper“, „Leib-Seele-Verhältnis“, „Geist“" und „Seele“ in Ritter/Gründer/Gabriel (Hg.) 1971-2007). Es kommt hier nur ,auf das populäre Bewußtsein“ (ebd., Bd. 2, Sp. 282) an. 
vorrangiger Modus aus, bleibt aber in nonverbale Modi (z.B. Gestik und Mimik) eingebettet. Dieser erste Kommunikationstyp heißt (sachlich nicht ganz zutreffend) unmittelbare Kommunikation, weil nur der menschliche Körper ohne technische Unterstützung als Medium dient. Er ist also MULTIMODAL und MONOMEDIAL.

2) Im Laufe der (Entwicklungs- wie Individual-)Geschichte wird diese ursprüngliche Kommunikationsform immer mehr ergänzt durch zunehmend abstrakte Kommunikationsformen: Elemente der ursprünglichen Situation (a) fallen weg und (b) werden (teilweise) durch technische Mittel ersetzt; „semiologisch integrierte Aktivität“ (Harris 2008, S. 18) wird also komplexer. (i) Kommunikation löst sich ab von der unmittelbaren Einbindung in arbeitsteilig-praktische Überlebensprozesse. Sie dient auch Magie, religiösen Praktiken und spielerischer Lebensfreude. (ii) Zunehmend wird Sprache auch als Reflexionsmittel benutzt. Philosophie, Mathematik und intellektuelle Berufe entstehen. ${ }^{10}$ (iii) In dieser Linie von Entsinnlichung und Vergeistigung entdeckten unsere Vorfahren vor wenigen Jahrtausenden mit der Erfindung von Schrift eine andere materielle und modale Grundlage von Sprache: Neben den akustischen tritt ein optischer Träger, statt Mund und Ohr fertigen Hand und Auge die Botschaft. ${ }^{11}$

\subsection{Zerdehnte Kommunikation}

Die neuen Übertragungsmittel sind nicht mehr flüchtig, sondern beständig. So erlaubt Schrift Kommunikation und Überlieferung unabhängig von Raum und Zeit, also - wie Ehlich (1983, S. 32) sagt - in einer „zerdehnten Sprechsituation“. Das hat natürlich Folgen für die sprachliche Form. Schriftsprache entsteht. Alles muss ausformuliert werden, was der Empfänger über die Situation des Senders nicht wissen kann, weil ein gemeinsamer Wahrnehmungsraum fehlt. Und weil nicht unmittelbar reagiert oder rückgefragt werden kann, muss kohärenter Sinn in kohäsiven Texten ausgeprägt werden, die als geschlossene Monologe und mehr oder weniger autonome Ganzheiten möglichst aus sich selbst verstanden werden können. Entsprechend werden grammatisch wohlgeformte Sätze in klarer innerer Gliederung und möglichst geregelten Mustern (Textsorten) erwartet. So kann man sagen, „daß mit der Erfindung der Schrift die Spezifik des Texts materialisiert wird.“ (ebd., S. 39) Und mittels Schrift können auch die Formen gesellschaftlichen Wissens anders organisiert werden, weil das Gedächtnis entlastet wird.

Zwar wird sowohl beim Verfertigen als auch beim Lesen schriftlicher Texte zuerst noch laut gelesen. Für die Antike resümiert Ludwig (2005, S. 72 f.) sogar: „Texte gibt es eigentlich nur als gesprochene.“ Doch von

\footnotetext{
$10 \quad$ Soweit vgl. Malinowski (1974).

11 Zur Evolution von Sprache insgesamt vgl. Leroi-Gourhan (1980).
} 
Anfang an unterstützt Schrift Reflexion, nicht zuletzt auch über Sprache selbst. Oder, wie Humboldt (1994c, S. 100) sagt: „Ihre allgemeinste Wirkung ist, dass sie die Sprache fest heftet, und dadurch ein ganz andres Nachdenken über dieselbe möglich macht."

Im Anschluss an Ehlichs Ausdruck nenne ich diesen zweiten Typ gesellschaftlicher Verständigung zerdehnte Kommunikation. Zerdehnte Kommunikation ist tendenziell MONOMODAL und MONOMEDIAL; prototypisch funktioniert sie über klar ausformulierte Ganztexte.

\subsection{Verdichtete Kommunikation}

Mit wachsender gesellschaftlicher Differenzierung wird dieser Typ immer komplexer, so dass daneben allmählich ein zusätzlicher dritter Typ heranwächst, den ich verdichtete Kommunikation nennen möchte. Zunächst, vor einem halben Jahrtausend, lässt die Erfindung des Buchdrucks ${ }^{12}$ als zweite kommunikationstechnische Revolution den zerdehnten Raum zwischen den Kommunikationspartnern auf völlig neuartige und vielfältige Weisen ausfüllen. Reichweite und Frequenz der Fernkommunikation werden erhöht, neue Funktionen entdeckt, für Materialien, Modi, Gestaltung und Formen der fixierten Botschaften ungeahnte Variationen entwickelt, und es entstehen neue schriftliche Standardsprachen, zugerichtet - wie Giesecke (2006, S. 489) sagt - „auf die technischen Parameter“ des Buchdrucks. ${ }^{13}$ Schrift steigt zum dominanten und angesehensten Kulturträger auf; ${ }^{14}$ Mündlichkeit und Bildlichkeit treten demgegenüber zurück.

Audiovisuelle Medien im 20. Jahrhundert dann (bis etwa 1990) erweitern das mediale Spektrum. Telefon (gesprochene Sprache), Schallplatte und Hörfunk (plus Ton und Musik), Film und Fernsehen (plus bewegte Bilder) technisieren immer mehr Kommunikationskanäle und untergraben allmählich die vorübergehende Monopolstellung der Schrift. Derart verdichtete Kommunikation muss nicht notwendig mehr zerdehnt, sondern kann teilweise synchron geschehen. Gleichzeitig wandern Zeichen aus individueller Verfügung heraus und treten Individuen massenhaft gegenüber fast als ein Lärm und Rauschen, in dem sinnvolle Orientierung eigener Kulturtechniken bedarf. (Man stelle sich dagegen vor, welch eine geringe Informationsmenge, wie wenig Wissen also ein Proto-Google in der Antike hätte verwalten müssen.) Verdichtete Kommunikation ist MULTIMODAL und MULTIMEDIAL.

\footnotetext{
12 Dazu Giesecke (2006 [1991]); vgl. auch Füssel (1999).

13 In gleicher Weise hat im 20. Jahrhundert der Hörfunk zur Durchsetzung einer überregionalen mündlichen Standardsprache beigetragen.

14 Luthers (1521, S. 98) später zum Schlagwort gewordene Wendung „solam scripturam regnare" trifft über den Bibelbezug hinaus einen Kern des kulturellen Wandels.
} 
Und dann, erst in unserer Generation, tritt mit dem hochentwickelten Computer eine semiotische Universalmaschine auf den Plan, die fast alles Kommunizierbare technisieren kann - auch wenn die Hardware (der materielle Körper) derzeit immer noch recht beschränkt daher kommt: viel Material, kleine, starre, schwere Bildschirme, träge Akkus, immer noch zahlreiche Kabel, umständliche Peripherie-Geräte. ${ }^{15}$ Über all das werden wir in 20 Jahren lachen. Freyermuth (2005, S. 19, 21 f.) hat zu Recht darauf hingewiesen, dass erst die digitale Technik eine wirkliche Verschmelzung der verschiedenen Medien ermöglicht und dass Hardware immer mehr durch Software ersetzt wird - mit der Folge, dass materieller Mangel, technische Beschränkungen und materiale Differenzen schwinden.

Für unser Thema spielt aber nur die Oberfläche eine Rolle, also die so genannte ,Schnittstelle zwischen Mensch und Maschine, insbesondere der Bildschirm. ${ }^{16}$ Computerkommunikation technisiert tendenziell alle Dimensionen der ursprünglichen Kommunikationssituation, macht also die bisherigen mediengeschichtlich erzwungenen Einschränkungen und daraus erwachsenden Abstraktionen bei der Technisierung natürlicher Kommunikation möglichst rückgängig: 1) Mit multimodalen Zeichen kann die Einbindung der Kommunikationspartner in eine mehr oder weniger gemeinsame Situation hergestellt (z.B. in Videokonferenzen) oder zumindest simuliert (z.B. in Chats) werden. 2) Das Maß an zeitlicher Zerdehnung kann beliebig manipuliert werden bis hin zur völligen Aufhebung in simultaner Online-Kommunikation. 3) Räumliche Distanzen werden kaum noch als solche erlebt. Je mehr Eigenschaften unmittelbarer Kommunikation (Typ I) technisierte Kommunikation sich anverwandeln kann, desto erfolgreicher wandert sie in Alltagskommunikation ein und durchzieht sie. Das ist das bestimmende Merkmal verdichteter Kommunikation (Typ III): Sämtliche Arten technisierter und unmittelbarer Kommunikation spielen gleichzeitig und hochkomplex eng zusammen.

Einerseits ist erstaunlich, dass die Evolution das noch mitmacht und die biologischen Möglichkeiten menschlicher Körper nicht an ihre Grenzen stoßen. Andererseits werden stets neue, aus der Sicht der alten oft hybride, Kommunikationsformen und Ausdrucksweisen erfunden, die den neuen Bedingungen möglichst gut angepasst sind ${ }^{17}$ - so auch die unten zu beschreibenden Spielarten von Schriftlichkeit.

15 „noch obsiegt der Widerstand der Materie über den menschlichen Willen“ - so begründet Stefan Zweig (1997, S. 153) den beschränkten Rhythmus menschlicher Bewegung bis weit ins 19. Jahrhundert hinein.

16 Ich denke hier nur an PCs und Laptops. Für die viel kleineren Multimedia-Handys wurden teils semiotisch schlankere Text-Bild-Kombinationen entwickelt.

17 Und - worauf Raab (2008, S. 45) hinweist - ,immer wieder Sehattraktionen bieten, welche die Wahrnehmung in neue, unbekannte Blickräume und Blickweisen überführen, die das Sehfeld über den Horizont hinaus erweitern und dem Betrachter immer wieder die Grenzenlosigkeit des Sichtbaren und noch zu Schauenden vor Augen führen.“ 
Für die Zukunft können wir also erwarten, dass Kommunikation noch dichter, komplexer und multimodaler wird. Digitalisierung macht technische Kommunikation immer flexibler und ihre materiellen Grundlagen weniger sperrig und weniger sichtbar. In der weitgehend schriftgebundenen Phase zerdehnter Kommunikation wanderten Zeichen aus unmittelbaren Situationen aus in scheinbar selbständige zweite Welten. Kien in seiner Bibliothek in Canettis Roman „Die Blendung“ (1936) steht emblematisch dafür. Im gegenwärtigen Zeitalter dichter Kommunikation wird der Bibliomane von Computersüchtigen in Second Worlds abgelöst; gleichzeitig durchdringt technisierte Kommunikation aber auch, viel stärker als früher, den ,normalen' Alltag und wächst in unmittelbare Kommunikation ein. Dabei verliert Schrift ihren viele Jahrhunderte währenden dominanten Status an einen multimodal-barocken Kommunikationsraum mit allerdings neuartigen, dezentralen Herrschaftsstrukturen. Er trägt und prägt die meisten Diskurse. Schriftlichkeit nimmt darin neue Funktionen und Formen an.

Das heißt mitnichten, dass Materialität verschwände, also in ,Immaterialität ${ }^{\dagger}$ aufginge. Doch die Art und Weise, wie wir unsere Vorstellungen in Zeichen materialisieren, folgt natürlich auch jetzt den neuen physikalischen Bedingungen.

\section{Res cogitans extensa}

Die skizzierte Entwicklung zunehmender Abstraktion und teilweise Technisierung von Elementen der ursprünglichen, unmittelbaren Kommunikation förderte unsere geistigen Abstraktionsfähigkeiten und schließlich -gewohnheiten. Empraktische Tätigkeit tritt, wie bei Kien, in den Hinter-, geistige Arbeit im Feld der Zeichen in den Vordergrund. Schon die berühmteste Darstellung der Ursprungssituation, Bühlers (1934, S. 102-120) Hier-jetzt-ich-Origo, abstrahiert stark von der dialogischen Wechselseitigkeit der Sprecher-Hörer-Rollen und argumentiert vornehmlich aus der egozentrischen Sicht eines der Partner. ${ }^{18}$ Ganz allgemein denken erwachsene Menschen weniger aus der direkten Begegnung mit materiellen Gegebenheiten heraus als viel mehr geistig vermittelt über Zeichen. Im Laufe der Menschheitsgeschichte bildete sich immer umfassender eine scheinbar eigenständige Welt aus Zeichen aus, durch die hindurch wir die materielle Realität wahrnehmen. ${ }^{19}$

18 Weinrich (1988, S. 82) weist auf die egocentric fallacy hin, der Bühler unter dem Einfluss von Fichtes idealistischer Philosophie erlegen sei. „Wenn Sprache nämlich in ihrer kommunikativen Natur an das Substrat des Leibes gebunden ist, kann sie nicht bei einem einsamen Ich ihren Ausgang nehmen, sondern hat als ihre origo die kommunikative Dyade, bestehend aus einem Sprecher/Hörer und einem Hörer/Sprecher, die beide kommunikativ gleichberechtigt sind."

19 Vgl. Peirce (1967, S. 42): „Wir haben kein Vermögen, ohne Zeichen zu denken.“ (CP 5.265). 
Dieser Verlauf unterstützte in dem Bild, das wir uns von unserer Welt machen, die Vorstellung von einer (dann leichtfertig als ontologisch gedachten) Zweiteilung/Dichotomie: Auf der einen Seite (unten) die res extensa (body, Materie; eher Hölle, unten, Hauptschule, Handwerker im blue collar), auf der anderen die res cogitans (mind/soul, Geist/Seele; eher Himmel, oben, Gymnasium, Wortwerker im white collar). Descartes, auf den die griffigen lateinischen Termini zurückgehen, sah das wesentlich differenzierter ${ }^{20}$ doch durchzieht die religiös untermauerte und dogmatisch trivialisierte Tradition den abendländischen Alltagsdiskurs. (Sie erinnern sich an das NespressoLogo unten rechts im vorigen Beispiel:)

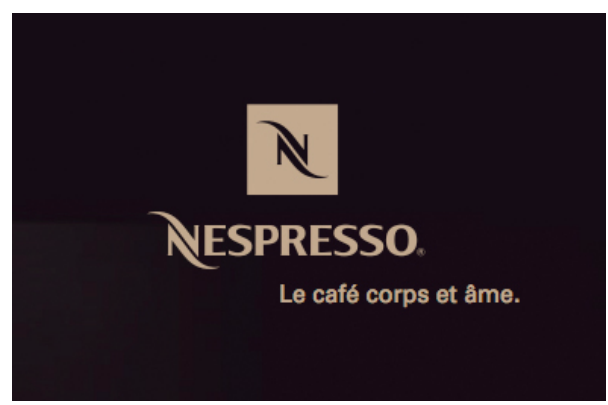

Abb. 2: Nespresso-Logo (Ausschnitt aus Abb. 1)

In Wirklichkeit gibt es keine res cogitans ohne extensa. Insbesondere - das möchte ich zeigen - prägt die materielle Grundlage die Form unserer Zeichen mit. Doch ein Großteil der linguistischen Theorie im 20. Jahrhundert bewegt sich im Diskurs über Sprache, wie er Typ II (zerdehnte Kommunikation zwecks möglichst unveränderter Aufbewahrung und Überlieferung im Botenmodell) ${ }^{21}$ entspricht. Darin kommt es ja auf den Wortlaut an, den identischen Sinn, der möglichst getreu zu übermitteln und zu verstehen ist. Und dafür spielt die abstrakte Norm die entscheidende Rolle; eigene Merkmale der äußeren Form, vor allem aber der materiellen Grundlage, sind in ihrer für diesen Zweck störenden Widerspenstigkeit möglichst zu neutralisieren. ${ }^{22}$ Durch Konzentration auf die Form ist (oder vielleicht: war?) Materialität, der ,Körper` unserer Mitteilungen, weitgehend aus dem Blickfeld der Sprachwissenschaft verschwunden - ähnlich wie der menschliche Körper aus dem Blickfeld der Mainstream-Psychologie geriet (Costall 2007, S. 56, 58-64). So wurden Aura, Stimme und Stimmung, Aussprache, Ak-

20 Vgl. etwa Costall (2007, S. 57 f.).

21 Dazu Krämer (2008).

22 Solches Denken beförderte ,a semiotics in which matter did not matter. [...] In disregarding matter in this way, linguists and semioticians followed a cultural trend in which the de-contextualised power of design reigned over the specifities, the practicalities and the realities of everyday life and art" (Kress/Van Leeuwen 2001, S. 69 f.). 
zent, Prosodie, Intonation, Gestik, Mimik, Proxemik, Handschrift und alle individuell-performativen Merkmale von Kommunikation marginalisiert oder psychologisiert. ${ }^{23}$

Man denke an das bilaterale Zeichenmodell der mittelalterlichen Scholastik, das über Saussures (1967) Cours wieder Einfluss gewann. ${ }^{24}$ Jede Verbindung von Ausdruck und Inhalt gilt als Zeichen. Der Strukturalismus konzentriert sich dabei auf die Form beider Seiten. In der eben skizzierten Tendenz, geistige von körperlichen Erscheinungen zu isolieren, werden materielle Grundlagen von Zeichen ignoriert. ${ }^{25}$ Eine vollständige Skizze hingegen müsste so aussehen:

\begin{tabular}{|c|c|c|c|}
\hline \multicolumn{3}{|c|}{ Verbindung durch sich bietende Gelegenheit oder bewusste Wahl } \\
\hline \multicolumn{2}{|c|}{ MATERIELLER TRÄGER } & \multicolumn{2}{|c|}{ GEISTIGE FORM } \\
\hline Rohstoff & Form & Ausdruck & Inhalt \\
\hline Verbindung durch körperliche Tätigkeit & \multicolumn{3}{|c|}{ Verbindung durch Konvention } \\
\hline
\end{tabular}

Wenn Menschen (ja stets durch Zeichenbildung) kommunizieren, prägen sie einem materiellen Träger (Schallwellen, Farbpigmenten u.a.) durch körperliche Tätigkeit Formen auf, die sie als konventionalen Ausdruck intentionaler Inhalte verstehen. Aus Natur wird Kultur. In günstiger Routine geschieht das reibungslos. Erst unter widrigen Umständen oder bei großer Kreativität wird die Spannung zwischen totem Rohstoff und lebendiger Mitteilung spürbar. Doch immer prägt die materielle Grundlage die Form des Ausdrucks mit.

Noch im Diskurs über „Materialität““ wird Material verleugnet. ${ }^{26}$ Der damals wegweisende, über 900 Seiten starke Sammelband von Gumbrecht/ Pfeiffer (Hg.) (1988) über „Materialität der Kommunikation“ kreist um „Se-

23 Vgl. dazu auch Koch/Krämer (Hg.) (1997), darin insbesondere die Einleitung der Herausgeber. Krämer (1997, S. 107 f.) erinnert in diesem Band u.a. auch daran, wie Derrida die abendländische Reflexion über Schrift „als Symptome einer mißglückten Verdrängung“ deutet: „Verdrängt werden dabei die für die Schrift konstitutiven Phänomene der Materialität, der Äußerlichkeit, der Abwesenheit und der Nicht-Ursprünglichkeit; das sind aber allesamt Phänomene, welche die philosophische Plausibilität einer Idealität des Sinns, einer Metaphysik der Präsenz und einer am intentionalen Bewußtsein orientierten Theorie des Geistes unterminieren könnten.“ - Zur Materialität von Schrift vgl. etwa Greber/Ehlich/Müller (Hg.) (2002).

24 „Ich nenne die Verbindung der Vorstellung mit dem Lautbild das Zeichen.“ (Saussure 1967, S. 78). Der authentische Saussure allerdings erfasst erheblich intensiver das performative Zeichenspiel; vgl. etwa Jäger (2008).

25 „Das sprachliche Zeichen ist also etwas im Geist tatsächlich Vorhandenes.“ (Saussure 1967, S. 78).

26 Etwa in der Art des Philosophenkongresses über die Frage, ob der Gelbe Fluss wirklich oder nur in den Köpfen existiert: Sämtliche Teilnehmer kamen durch eine Überschwemmung ums Leben, weshalb die Frage bis heute „ungelöst“ blieb (Brecht 1967, S. 2211 f.). 
mantiken von Materialität" (Pfeiffer 1988, S. 17) und beschäftigt sich gleichermaßen mit dem menschlichen Körper bzw. Leib, mit Apparaten, Medien und materiellen Trägern von Kommunikation als selbst (angeblich) nicht sinnhaften Voraussetzungen von Sinn. Ich meine mit ,Materialität ${ }^{27}$ hier nicht das Reden über derlei unterschiedliche Dinge, sondern viel nüchterner und ganz einfach die stoffliche Grundlage von Zeichen, das Material, mit dem wir Zeichen bauen, also den physikalischen Träger von Sinn. Das wirft die simple Frage auf: Was macht die Tinte (oder eben das Pixel) mit dem Sinn? ${ }^{28}$ Führt, zum Beispiel, Handschrift zu anderen Texten - hier Thomas von Aquin -

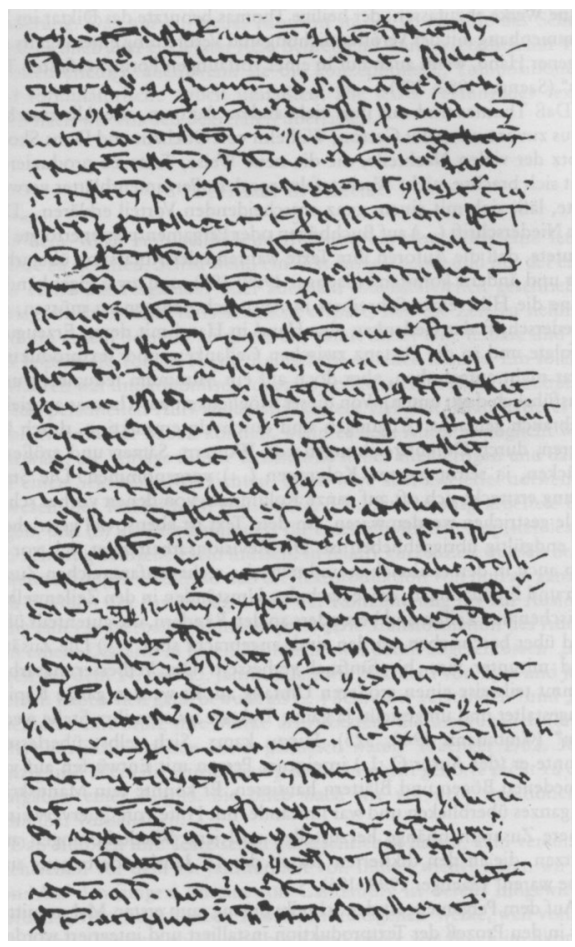

Abb. 3: Thomas von Aquins Handschrift ${ }^{29}$

$27 \quad$ Zur wechselvollen Geschichte des „Materie“-Begriffs siehe Detel et al. (1980).

28 Krämer (2008, S. 295) meint, in metaphysischer Sicht erscheine die Unmittelbarkeit des materiellen Zeichenträgers als „Oberfläche, die es zu durchdringen gilt, um zu dem nicht mehr sichtbaren, vielmehr nur noch interpretierbaren Zeichensinn zu gelangen“. Beim Medium dagegen verhalte es sich genau umgekehrt: „Die Unmittelbarkeit des medial zur Erscheinung kommenden Sinns bildet demgegenüber eine Oberfläche, die es zurückzulassen gilt, um in ihrer $>$ Tiefe $<$ erst die verborgene Materialität des Mediums freizulegen." Das scheint mir wirklich sehr metaphysisch.

29 Ludwig (2005, S. 177), dort mit Quellenangabe. 
als Mündlichkeit, SMS, Chat oder ein Textverarbeitungsprogramm; und erzeugen unterschiedliche technische Plattformen unterschiedliche performative Effekte? (Hier US-Präsident Obamas Unterschrift:)

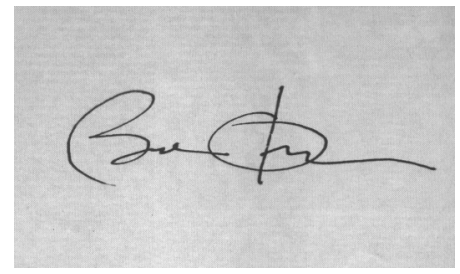

Abb. 4: Barack Obamas Unterschrift ${ }^{30}$

Um solche Fragen ${ }^{31}$ zu beantworten, müssen wir zunächst drei variable Rahmenbedingungen erörtern, welche menschliche Kommunikation in entscheidender Weise tragen und prägen, nämlich die stoffliche Grundlage der Zeichen (Material), das genutzte Kommunikationsgerät (Medium) und die situativ bzw. medial ermöglichte kommunikative Konstellation (Kommunikationsform).

\section{Rahmenbedingungen menschlicher Kommunikation}

\subsection{Material - Medium - Kommunikationsform}

In Auseinandersetzung mit ihren Lebensbedingungen schaffen sich Menschen das, was sie brauchen. (Piaget (z.B. 1975) beschrieb das als Wechselspiel von Assimilation und Akkommodation.) So auch Sprache: „Das Bedürfnis schuf sich sein Organ“ (Engels 1962, S. 446). Und ihre Erzeugnisse wirken wieder auf sie zurück. Das gilt auch für Veränderungen im Kommunikationsgefüge. Wachsende gesellschaftliche Bedürfnisse treiben Menschen dazu an, ihre Kommunikationsbedingungen zu bearbeiten, um kommunikative Potentiale zu erweitern. Zu diesen Bedingungen zählen insbesondere Material, Medium und Kommunikationsform. ${ }^{32}$ Im aktuellen Stadium dichter Kommunikation treiben drei Neuerungen die Entwicklung maßgeblich voran: das Material Pixel, das Medium Bildschirm und die

\footnotetext{
30 Frankfurter Allgemeine Zeitung, 22.1.2009, S. 1.
}

31 Schnell gesellen sich verwandte hinzu, zum Beispiel (um zwei schriftbezogene Extreme zu nennen): Was geschieht auf dem Weg von der Dichterhandschrift zur kritischen Edition (etwa Hölderlin 1975-2008), was bei der Verschriftung von Mündlichkeit (dazu etwa Ingenhoff/Schmitz 2000)? Dieser Aufsatz konzentriert sich auf eine Fallgruppe (Pixelschrift an Pixelbild) in der Hoffnung auf möglichst genaue und partiell dennoch exemplarische Teilantworten.

32 Der begrifflichen Klarheit und intellektuellen Fruchtbarkeit halber unterscheide ich bewusst zwischen diesen dreien (stoffliche Grundlage von Zeichen, Kommunikationsgerät und situativ bzw. medial ermöglichte kommunikative Konstellation). Diese Unterscheidung wird nicht allgemein anerkannt; zur Diskussion vgl. Schneider (2008, S. 243 f. (Anm. 245)). 
Kommunikationsform Hypermedia (insbesondere World Wide Web). Gemeinsam tragen sie dazu bei, dass viele der klassischen Vorteile unmittelbarer Kommunikation technisch wenigstens teilweise simuliert und einschränkende Reduktionen zerdehnter Kommunikation partiell aufgehoben werden können. Das verändert auch Schriftlichkeit.

Betrachten wir zunächst ein konkretes Beispiel (Kap. 4.2) und widmen uns anschließend den drei genannten Faktoren in abstrakterer Weise (Kap. 4.3 bis 4.5).

\subsection{Drittes Beispiel: Komponierte Schriftfragmente}

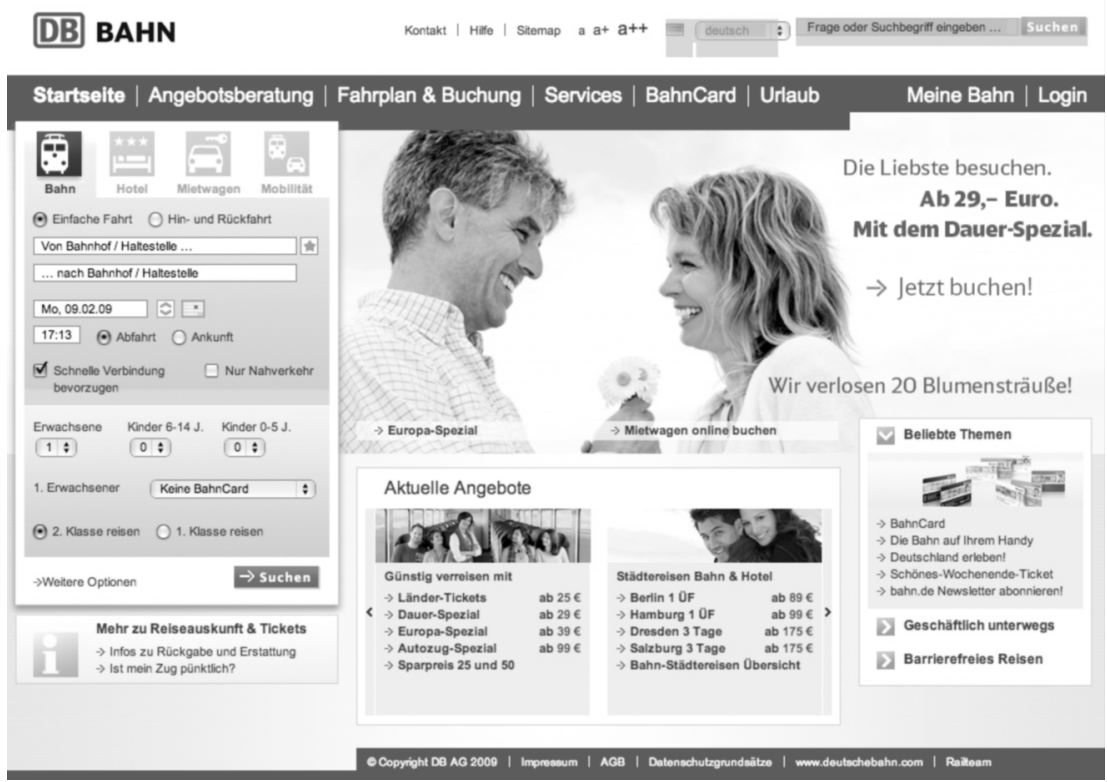

Abb. 5: bahn.de (www.bahn.de (Stand: 9.2.2009))

Verglichen mit dem (vorigen) Clooney-Beispiel (Abb. 1) enthält dieses sehr viel mehr Text. Die Bilder dienen nur zur Dekoration und laden den Text kaum oder gar nicht auf. Doch das Layout, also die Platzierung und visuelle Gestaltung und Begleitung des Textes auf der Fläche, ist für sein Verständnis unerlässlich. Als fortlaufender Fließtext ließe er sich nicht entschlüsseln. Das Layout, nicht Grammatik, sorgt für Gliederung und Kohäsion. Ansatzweise war das auch bei herkömmlichen Papierformularen schon so. Doch auf dem hypermedialen Bildschirm übernehmen (teilweise konventionalisierte) Flächenverteilung, Farbgebung, unterschiedlich gestaltete Kästchen und Piktogramme viel mehr Anteile der Botschaft, so dass der wohlplatzierte Text noch ganz erheblich kürzer werden kann. Man betrachte etwa 
das linke (knappe) Drittel von Beispiel 3. Dort zählen wir 48 Wörter (25 davon sind Substantive), drei Abkürzungen sowie einige Ziffern, Zahlen und 18 Interpunktionszeichen. Außer beim Vierwortsatz in der letzten Zeile beschränkt sich Syntax auf die lineare Anordnung der Elemente. Leicht flektiert werden nur je drei Substantive und Adjektive im Nominativ; Kasus spielen keine Rolle. Artikel und Adverbien kommen nicht vor. Material, Medium und Kommunikationsform ziehen eine Schriftlichkeit nach sich, die mit den gewohnten klassischen Formen wenig gemein hat.

\subsection{Material Pixel}

Das Wort Pixel ist eine Kreuzung aus englisch pictures (kurz pix) und element und bezeichnet eben als kleinste Bildeinheit einen Bildpunkt, nämlich die kleinste Einheit einer digitalen Rastergrafik, wie sie heute auf Bildschirmen dargestellt wird. Gutenbergs mobile Lettern erlaubten die bis dahin unvorstellbare massenhafte Reproduktion von Texten, indem sie den schriftlichen Wortlaut in kleinste Einheiten des Druckens zerteilen ließen. Diese Lettern mussten allerdings zu Hunderttausenden handgreiflicher Werkstücke teuer gegossen werden. Die viel winzigeren Pixel hingegen treiben Gutenbergs Modularisierungs-Idee auf die Spitze. Sie können in praktisch beliebig gigantischer Anzahl fast kostenlos hergestellt werden. Thre stoffliche Grundlage ist minimal, sie sind von jeder Bindung an semiotische Systeme (z.B. Buchstaben) befreit und können eben deshalb alle visuellen Kommunikationsmodi gleichermaßen darstellen. ${ }^{33}$ Bei hinreichend feiner Körnigkeit verliert dabei der technische Gegensatz von analoger und digitaler Signalverarbeitung seine Bedeutung für die menschliche Wahrnehmung. ${ }^{34}$

Weil jedes klitzekleine Pixel einzeln manipuliert werden kann, verschwindet auch der technisch bedingte Unterschied zwischen Text und Bild; und in beiden Modi werden neue Potentiale freigesetzt. Anfang 2009 wurden 14 weltberühmte Gemälde aus dem Prado im Internet zugänglich gemacht, und zwar jeweils als Foto aus rund 14.000 Megapixeln..$^{35}$ Das heißt, die Fläche eines Gemäldes wird auf 14 Milliarden Pixel verteilt (eintausendvierhundert Mal so viel wie bei einer modernen Digitalkamera). Das erlaubt nicht nur unendliche Reproduktion an beliebigem Ort zu beliebiger

\footnotetext{
33 Pixel setzen digitale Informationen in Erscheinungsformen um, die von menschlichen Sinnesorganen wahrgenommen werden können. Mit entsprechender Soft- und Hardware können digitale Informationen physikalisch auch anders realisiert werden, so dass multimodale Botschaften auf ein und derselben technischen Plattform übermittelt werden können. In diesem Beitrag geht es aber nur um Schrift an und als Bild.

34 Insoweit wird auch der oft betonte Gegensatz zwischen kontinuierlichen (etwa mündlichen) und disjunktiven (etwa schriftlichen) Kommunikationsmitteln hinfällig. Darüber wundert sich schon Fischer (1997, S. 98), zumal es seine äußerst intelligenten Beobachtungen über Schrift als Notation teilweise in Frage stellt.

35 www.google.com/prado (Stand: 17.1.2009).
} 
Zeit, sondern auch eine völlig andere Wahrnehmung. Man kann wie mit der Lupe in kleinste Details sehen, die das bloße Auge vor dem Original nicht wahrnähme.

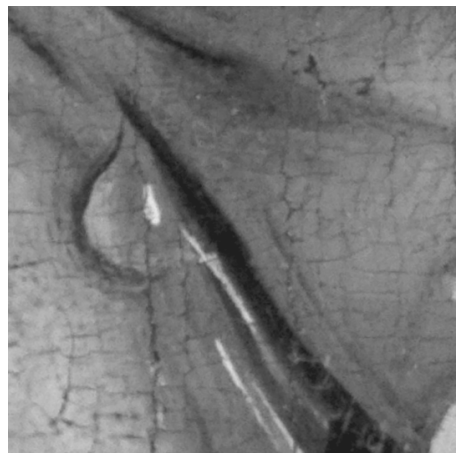

Abb. 6: Roger van der Weyden: Kreuzabnahme (ca. 1435) (Ausschnitt Träne)

Der Betrachter erhält ganz andere Eindrücke von den Maltechniken, den Proportionen, den Verhältnissen zwischen Detail und Ganzem. Digitalisierung verändert Produkt und Blick schon bei alten, handgemalten Bildern. Welch völlig neuartige Bilder erst können aus einzeln steuerbaren Pixeln angefertigt werden! Und wie verändert sich Schrift als Bild!?

Indem Pixel die materiellen Grundlagen von Zeichen miniaturisieren, entstofflichen (,virtualisieren') sie sie scheinbar. Das erleichtert und beschleunigt die sonst ja doch schwerfällige technische Kommunikation. Wenn Stoff zum Ausdruck von Zeichen verwendet wird, soll er als (eigentlich hinderlicher) Stoff (außer in künstlerischer Subversion) nicht mehr wahrgenommen werden. So verschwinden auch die einzelnen Pixel im Gesamtbild. In dem Maße, wie sie etwa bei vielfacher Vergrößerung in ihrer Körnigkeit als Material (physische Grundlage) sichtbar werden, tritt der Sinn, wie wir am Prado-Beispiel sahen, zurück. Dass der materielle Zeichenträger aber bis in Mikrodimensionen hinein manipuliert werden kann, die dem bloßen Auge nicht mehr zugänglich sind, eröffnet fast unbegrenzte Möglichkeiten, Zeichen nach Belieben zu bilden und formen.

\subsection{Medium Bildschirm}

Medien seien hier als Kommunikationsgeräte verstanden, also als technische Hilfsmittel der Kommunikation. ${ }^{36}$ Bildschirme, die farbig leuchtenden

36 Zur Vieldeutigkeit des Medienbegriffs vgl. Habscheid (2000), zur Begriffsgeschichte Hoffmann (2002). - Weite Mediendefinitionen gehen, auch bei Schneider (2006; z.B. S. 83-86), stets mit begrifflichen Vagheiten einher, die theoretische Unklarheiten oder Widersprüche erzeugen. Schneiders Argumente gegen die Fassung von ,Medien`als Kommunikationsgeräten scheinen mir hinfällig, wenn man in einer performanzorientierten Perspektive neben Medien auch Modi der Kommunikation berücksichtigt, nämlich visuelle (mimisch, gestisch, schriftlich, bildlich, ...), auditive (mündlich, tonal, ...), taktile etc. 
Fenster ins Web, sind unhandlich, schwer und bieten (meist) nur eine recht kleine Fläche. ${ }^{37}$ Anders als auf Papier wird deren Inhalt zwar ständig geändert, doch lokal auch vergessen: Sie liefern nichts als perfektionierte $\mathrm{Pa}-$ limpseste. Das potenziert, beschleunigt, fragmentiert und dezentriert Kommunikation. Die innere (semiotische) Beweglichkeit wird noch erkauft mit äußerer (materieller) Schwerfälligkeit. Ganz im Kontrast zur virtuos-virtuellen Schwerelosigkeit der Inhalte haftet noch zu viel wuchtige Materie am Gerät, und die jeweils aktuelle Sehfläche ist vergleichsweise winzig. Der Bildschirm ist der Engpass im Zugang zum Web. Das erzwingt besondere Präsentationsweisen, nämlich schnell überschaubare Fragmente in Modulbauweise zur schnellen Selbstmontage (siehe Kap. 5).

\subsection{Kommunikationsform WWW}

Das World Wide Web ist die universalste, dichteste und reichhaltigste technische Kommunikationsform. ${ }^{38}$ Eine von beliebig vielen Institutionen und Personen bereit gestellte, grundsätzlich unendlich große, endlos erweiterbare und durch Hyperlinks beliebig untereinander vernetzbare Menge unterschiedlichster Informationen in sämtlichen digitalisierbaren Modi steht über Computer und ggf. andere Technologien (z.B. Mobiltelefone) allen Menschen auf der Welt zur Verfügung, die Zugang dazu haben (derzeit weit über eine Milliarde). ${ }^{39}$ Der fast grenzenlose Umgang mit Informationen aller Art erlaubt früher ungeahnte Möglichkeiten, macht es aber auch schwer, sich darin zurechtzufinden. Eines von mehreren Mitteln, Orientierungsverlust (lost in hyperspace) und kognitiver Überforderung (cognitive overload) entgegenzuwirken, ist ein besonderer Umgang mit Schrift. Und darauf gehe ich nun ein.

\section{Schrift für Blicke}

\subsection{Tertiäre Schriftlichkeit}

Die alte Kulturtechnik Schreiben hat oft ihre Funktionen, Materialien, Mittel, Formen und Erscheinungsweisen verändert. ${ }^{40}$ Mit der neuen Konstella-

37 Schon für Schrift auf Papier betont Mon (1995, S. 9), wie wichtig „,das format des spielraums und der lichtwert der druckbilder“ seien, „weil sich auf diese weise lesewerte über den bloBen wortsinn hinaus verkörpern."

38 Kommunikationsformen sind ,allein durch situative bzw. mediale Merkmale definiert, in kommunikativ-funktionaler Hinsicht also nicht festgelegt" (Brinker 2001, S. 139).

392007 fand Google über eine Billiarde Seiten (einzelne URLs), doch „Strictly speaking, the number of pages out there is infinite." (http://googleblog.blogspot.com/2008/07/we-knew-webwas-big.html (Stand: 6.2.2009). Internet-Statistiken: www.studeo.de/imafdi/statistik.htm (Stand: 6.2.2009).

40 Vgl. die einschlägigen Artikel in Günther/Ludwig (Hg.) (1994) sowie Ludwig (2005). 
tion von Pixeln auf Bildschirmen als Zugang zum hypermedialen WWW tritt wieder etwas Neues auf. Schrift - anders als gesprochene Sprache - war zwar immer schon visuell. ${ }^{41}$

Daß Sprache eine sinnlich wahrnehmbare Gestalt hat, daß das Bild durch Hinzufügung von Wortelementen und Schrift eine zusätzliche, gezielte geistige Dimension erhalten kann, wußten schon die frühesten Künste. (Weiss 1984, S. 7) (2 $^{42}$

Und schon (oder erst) im frühen Mittelalter wurden Texte so gegliedert, dass man „eine visuelle Vorstellung“ von ihnen bekommen konnte (Ludwig 2005, S. 119, vgl. S. 127 f.). Doch heute, im Zeitalter dichter Kommunikation und digitaler Techniken, metamorphiert Schrift selbst zum Bild. ${ }^{43}$ Eine neue Etappe von Schriftlichkeit (mit Vorläufern in alten Medien) kommt zur Blüte.

In Fortführung von Ongs (1987, S. 135-137) Unterscheidung in primäre und sekundäre Mündlichkeit kann man drei grundsätzlich verschiedene Arten von Schriftlichkeit unterscheiden (Schmitz 2006a, b). Klassische Schriftlichkeit in ihrer prototypischen Form (z.B. in Briefen, Protokollen oder Romanen) kommt monomodal (also rein schriftlich) daher, fixiert Sprache als mehr oder weniger endgültigen Text, dient zerdehnter Kommunikation und ist entsprechend auf situationsentbundene Abstraktion ausgerichtet sowie auf „analytische Reflektivität“ (Ong 1987, S. 136), geprägt von inhaltlicher Planung, sorgfältiger Form und innerer Abgeschlossenheit (autonomer Kohäsion und Kohärenz). Weitgehend im Gegensatz dazu entwickelt sich heute, im Zeitalter digitaler Medien, (z.B. in Chats) eine halbspontane (ich nenne sie sekundäre) Schriftlichkeit, die einige Merkmale kultiviert oder simuliert, die man sonst aus mündlicher Kommunikation gewohnt ist (vgl. z.B. Dürscheid 2003; Storrer 2001; Thaler 2005). An sehr viel ältere Vorläufer hingegen (z.B. in manchen mittelalterlichen Handschriften ${ }^{44}$ in Werbeplakaten und Illustrierten) knüpft eine in dieser Art ebenfalls neue, dritte Art von Schriftlichkeit mit eigenen Formen an. Sie

41 Grundsätzlich dazu Krämer (2003).

42 Unterschiedlichste „Formen visueller Textgestaltung oder Text-Bilder tauchen seit der hellenistischen Zeit in der Literaturgeschichte zu fast allen Zeiten immer wieder auf." (Weiss 1984, S. 17)

43 Wie so oft ist Kunst auch hier Vorreiterin. So bemerkt Weiss (1984, S. 13) über Concept Art und moderne Literatur im 20. Jahrhundert: „Es entstehen Texte, die zwischen Bild und Sprache nicht zu trennen brauchen, sondern ihre Aussagekraft aus beiden Medien beziehen. Die Schreib- bzw. Lese-Fläche ist zur eigentlich syntaktischen Dimension des Textes geworden.“ - Daraus folgt nicht, dass eine ,Logik des Schreibens“ einer ,Logik des Bildes“ unterworfen würde, wie Kress (2003, S. 163 f., passim) meint; zur Diskussion Schmitz (2006b, S. $84-189$ ff.).

44 Vgl. z.B. Ernst (2003). Im frühen Mittelalter werden erstmals bildhafte Elemente so in geschriebene Texte hinein verlegt, ,dass sie dort einen anderen Sinn erhielten“ (Ludwig 2005, S. 101). Bei tertiärer Schriftlichkeit heute verhält es sich teilweise genau umgekehrt; vor allem aber wird die gesamte Formenskala zwischen bildnahen und schriftnahen Elementen ausgenutzt. 
tritt überall dort auf, wo die visuelle Erscheinung und Umgebung von Schrift eine Rolle für ihr Verständnis spielt, insbesondere dort, wo Texte und Bilder auf ein und derselben Sehfläche platziert werden.

Heute sind die meisten visuellen Botschaften in öffentlicher Kommunikation als Sehflächen gestaltet, auf denen Layout, Bilder, Texte, Wörter und andere sichtbare Zeichen komplexe Beziehungen untereinander eingehen. Unter solchen Bedingungen ändert sich Schriftlichkeit selbst. Ihre optische Erscheinungsform wird nicht nur zu dekorativ-ästhetischen Zwecken, sondern in bedeutungsvoller Absicht gestaltet; sie nimmt einige Eigenschaften von Bildern an, geht Koalitionen mit ihnen ein oder ganz in ihnen auf; und sie ändert ihre innere Form von hierarchischer zur modularer Organisation. Tertiäre Schriftlichkeit nenne ich also solche, die Schrift als nichtautonomes Element in multimodalen Kontexten, vor allem in Text-Bild-Gefügen erscheinen lässt. Sie kommt in vielen Kommunikationsformen vor, von Texten an Waren über Plakatierung und Beschilderung im öffentlichen Raum bis zu Presse und anderen Papiermedien, am prägnantesten jedoch im World Wide Web. Denn nur auf Computerbildschirmen verschwindet der bisher (außer bei Handschrift) stets radikale technische Unterschied zwischen schriftlichen Texten und Bildern: Beide werden mit genau den gleichen technischen Mitteln und Einheiten dargestellt, nämlich mit beliebig steuerbaren einzelnen Pixeln.

\subsection{Viertes Beispiel: Pixeltext}
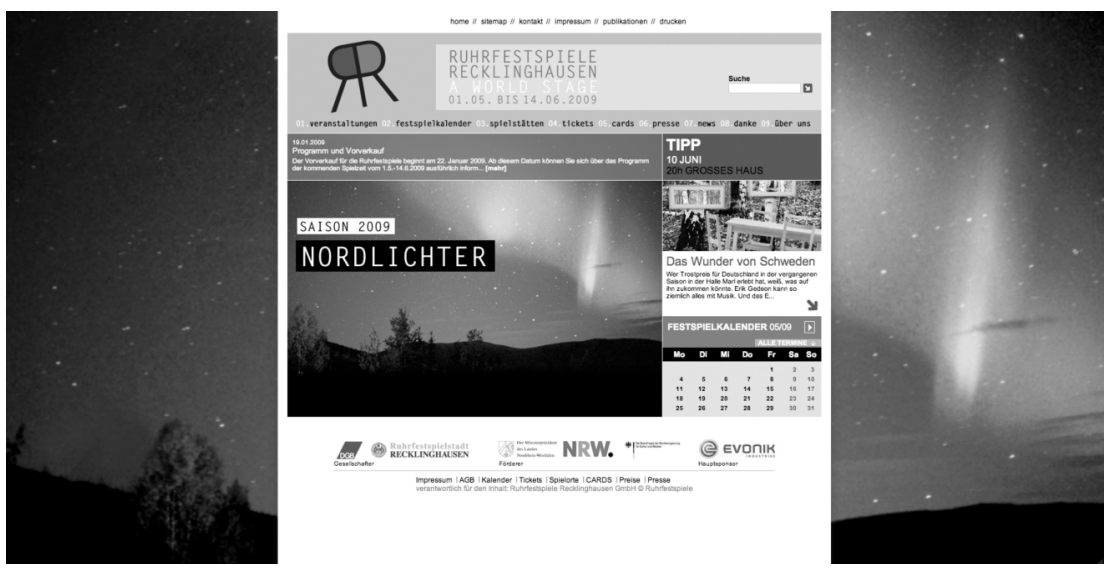

Abb. 7: Ruhrfestspiele (a) (www.ruhrfestspiele.de (Stand: 9.2.2009))

Auf einer Nordlichtlandschaft scheint ein bedrucktes Papier zu liegen, das jenes Hintergrundfoto wiederholt. (Spiel mit den Medien: Bild im Bild, simuliertes Papier auf dem Bildschirm.) Dieses vermeintliche Blatt enthält eine hybride Mischung aus Plakat, Pinnwand, Flyer, Werbelogos, Fragment eines Zeitungsartikels, Kalender und allerlei Hyperlinkbeschriftungen. 


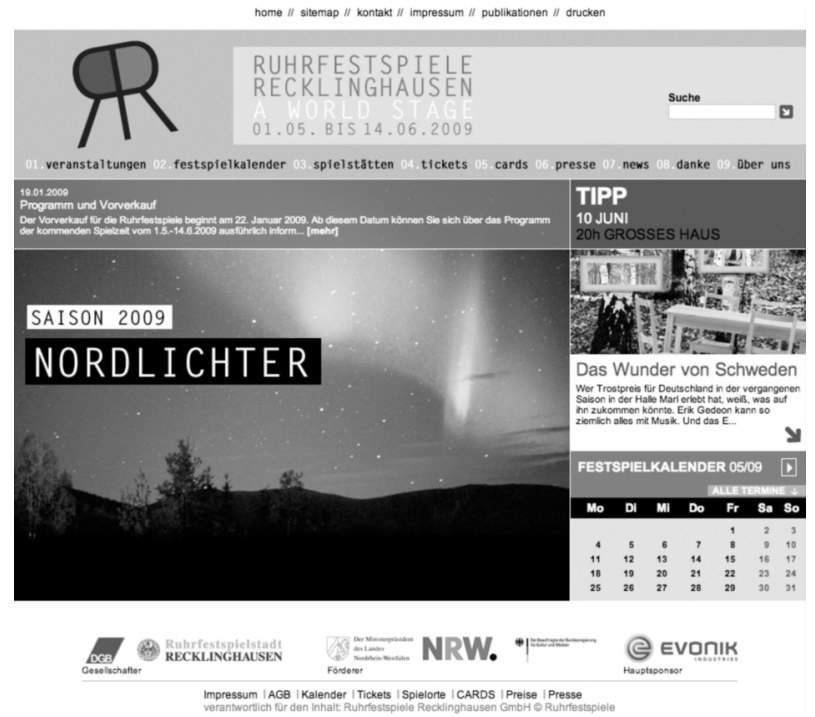

Abb. 8: Ruhrfestspiele (b) (www.ruhrfestspiele.de (Stand: 9.2.2009))

Die zahlreichen Einzelelemente werden allein übers Design zu einer kohärenten Gesamtbotschaft zusammengehalten, die beim ersten Blick verstanden wird etwa als ,Alles über die Ruhrfestspiele'. Trotz eines erheblichen Textanteils dominiert der visuelle Gesamteindruck samt (mehr dekorativen und suggestiven als informativen) Bildern und Seitengestaltung. Es gibt keinen einzigen vollständigen zusammenhängenden Text, sondern nur Fragmente, die wie Paratexte ${ }^{45}$ erscheinen, ohne sich jedoch auf einen Haupttext zu beziehen. Diese Elemente können großenteils nur dank ihrer Platzierung im Gesamtbild verstanden werden. Als fortlaufend gelesene Buchstabenkette ergeben sie keinen Sinn. Weil (mit zwei Ausnahmen) die kleinen sprachlichen Module nicht zu größeren sprachlichen Einheiten zusammengebaut werden, bedarf es keiner Grammatik; deren restliche Spuren (vor allem in Pluralmorphemen) regulieren Wortbildung und nicht Syntax.

Auch die beiden längsten Texte bleiben Fragment. Beide brechen, kaum begonnen, mit drei Punkten ab und erweisen sich als Türöffner für Texte hinter den Links. „Programm und Vorverkauf“ (weiß auf blau oben links) führt zur Wiederholung des gleichen Textes (schwarz auf weiß) und einen einzigen Satz mit 9 Wörtern. ${ }^{46}$ „Das Wunder von Schweden“ (rot auf weiß rechts in der Mitte) dient als Appetitanreger für einen Text hinter dem roten Pfeil, der herkömmliche Kohäsion und Kohärenz gerade nicht anstrebt, sondern die Botschaft in fast beliebig verteilbare Bauklötzchen zerlegt:

\footnotetext{
45 Paratexte sind einladend, beschreibend, orientierend, kommentierend oder ergänzend begleitende Nebentexte zu Haupttexten. Detailliert - allerdings nur für Bücher - Genette (1989).

46 „Das komplette Programmheft können Sie sich unter Publikationen herunterladen.“
} 


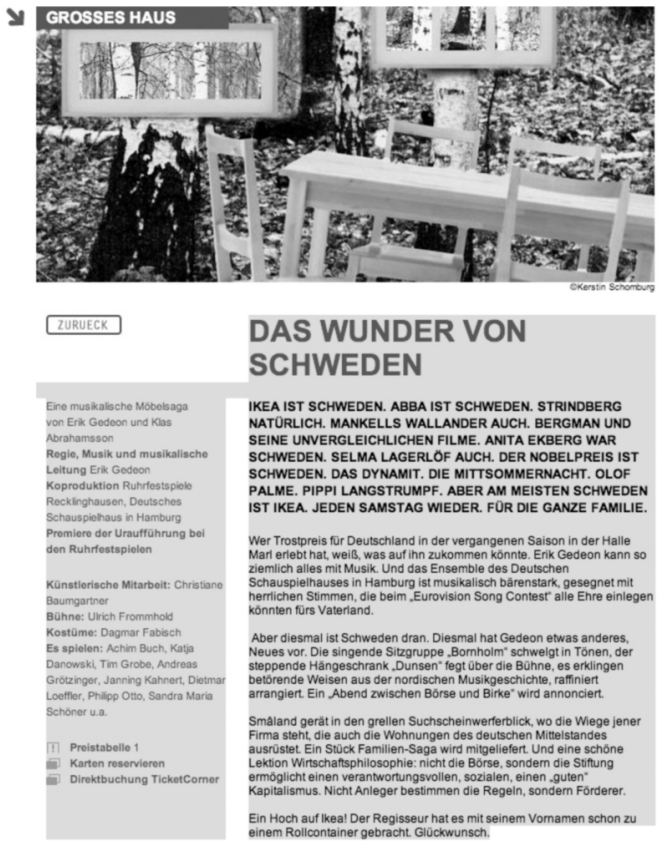

Abb. 9: Ruhrfestspiele (c) (www.ruhrfestspiele.de/veranstaltungen/veranstaltung detail.php?ver_id=30\&ter_id=121 (Stand: 9.2.2009))

Ikea ist Schweden. Abba ist Schweden. Strindberg natürlich. Mankells Wallander auch. Bergman und seine unvergleichlichen Filme. Anita Ekberg war Schweden. Selma Lagerlöf auch. Der Nobelpreis ist Schweden. Das Dynamit. Die Mittsommernacht. Olof Palme. Pippi Langstrumpf. Aber am meisten Schweden ist Ikea. Jeden Samstag wieder. Für die ganze Familie.

Wer Trostpreis für Deutschland in der vergangenen Saison in der Halle Marl erlebt hat, weiß, was auf ihn zukommen könnte. Erik Gedeon kann so ziemlich alles mit Musik. Und das Ensemble des Deutschen Schauspielhauses in Hamburg ist musikalisch bärenstark, gesegnet mit herrlichen Stimmen, die beim „Eurovision Song Contest" alle Ehre einlegen könnten fürs Vaterland.

Aber diesmal ist Schweden dran. Diesmal hat Gedeon etwas anderes, Neues vor. Die singende Sitzgruppe „Bornholm“ schwelgt in Tönen, der steppende Hängeschrank „Dunsen“ fegt über die Bühne, es erklingen betörende Weisen aus der nordischen Musikgeschichte, raffiniert arrangiert. Ein „Abend zwischen Börse und Birke" wird annonciert.

Smâland gerät in den grellen Suchscheinwerferblick, wo die Wiege jener Firma steht, die auch die Wohnungen des deutschen Mittelstandes ausrüstet. Ein Stück Familien-Saga wird mitgeliefert. Und eine schöne Lektion Wirtschaftsphilosophie: nicht die Börse, sondern die Stiftung ermöglicht einen verantwortungsvollen, sozialen, einen ,guten“ Kapitalismus. Nicht Anleger bestimmen die Regeln, sondern Förderer.

Ein Hoch auf Ikea! Der Regisseur hat es mit seinem Vornamen schon zu einem Rollcontainer gebracht. Glückwunsch. 
Da werden 214 Wörter auf 29 Sätze verteilt (Durchschnittslänge 7,4 Wörter), davon 12 Ellipsen und 7 Sätze mit dem Hauptverb „ist“ oder „war“. Von den restlichen 10 Sätzen (Durchschnittslänge 13 Wörter) sind sechs kurze einfache Hauptsätze, zwei Hypotaxen und nur zwei jeweils dreiteilige Satzgefüge. Der gesamte Text enthält sehr wenige explizit kohäsive Mittel (z.B. Rekurrenzen, Pronomen), die dann auch nur über sehr kurze Strecken wirken. Wer den Text als kohärent verstehen möchte, muss viel Vorwissen über die einschlägige Kulturszene sowie über Ikea aktivieren. Er will aber gar nicht als kohärenter Ganztext gelesen werden, sondern reiht einzelne Gedankenfetzen assoziativ und in fast beliebiger Folge um das hinter „Schweden“ versteckte Leitmotiv Ikea: eher Sprachcluster für selektive Wahrnehmung als linearer Text für sinnsuchende Lektüre.

Auf diese Weise unterwirft sich sogar der vergleichsweise lange Text, der ohnehin erst bei Aufruf durch Mausklick sichtbar wird, den streifenden Rezeptionsgewohnheiten für Bilder. So werden auch Texte „schnelle Schüsse ins Gehirn“, wie Kroeber-Riel (1993, S. ix) das für Bilder meint.

\subsection{Grammatik und Design}

Bilder werden vom ersten Gesamteindruck einer ganzen Gestalt zu den Teilen hin wahrgenommen, in die man bei genauerer Betrachtung dann näher eindringen kann. Bei herkömmlichen (längeren) Texten verhält es sich umgekehrt: Kleinere Teile (z.B. Wörter und Satzglieder) werden nach und nach zu größeren Ganzen (z.B. Teilsätzen und Sätzen) gefügt. Diesen Lektüreprozess unterstützt der Schreiber durch konventionelle Kohäsionsbildung. In monomodaler (primärer) Schriftlichkeit konnte er dabei zunächst notgedrungen allein auf grammatische Mittel zurückgreifen. Erst nach und nach wurde die physikalische Tatsache, dass Schrift visuell wahrgenommen wird, für die Gestaltung eines Schriftbildes ausgenutzt: Wortzwischenräume (Spatien), Interpunktion, Unterscheidung von Majuskeln und Minuskeln sowie immer weitere typographische und layouterische Mittel wurden entdeckt. ${ }^{47}$ Der Dichter Franz Mon (1995, S. 9) erinnert daran, „daß lesen ein umfassenderer vorgang ist als das entziffern von buchstaben, das ordnen fixierter zeichen zu einem sinn. [...] ein blatt papier, von ein paar wasserspritzern getroffen, ist bereits lesezusammenhang." Erst in tertiärer (bimodaler ${ }^{48}$ Schriftlichkeit jedoch verschmelzen Text und Bild zu gemeinsam getragenen Botschaften. Nutzer nehmen derartige Botschaften (z.B. auf Webseiten) eher als Bild denn als klassischen (monomodalen) Text wahr. ${ }^{49}$

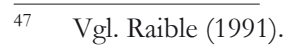

48 Bimodal-tertiäre Schriftlichkeit kann auch von weiteren (z.B. akustischen) Modi begleitet werden. Das scheint zwar ihre Rezeption, kaum aber ihre Struktur zu ändern und kann an dieser Stelle nicht näher untersucht werden.

49 Vgl. „Web users tend to scan pages, rather than read them.“ (Gahran 2002).
} 
Als Leser dieses Aufsatzes haben Sie das eben in den Abbildungen 7 und 8 selbst erlebt; sehr viel authentischer wirkt natürlich ein Blick auf diese oder andere Originalseiten am Bildschirm im Web.

So werden Kohäsion und Kohärenz auf solchen Sehflächen auch mehr durch visuelle als durch sprachliche Mittel erzielt. Die auf Anhieb (top down) erblickte Gestalt steuert die Wahrnehmung eher und stärker als ein zeitlich nach und nach (bottom up) aufgebauter Wortlaut. Fläche dominiert Linie; Design hat Vorrang vor Grammatik und macht sie (wie in den Abb. 5, 7 und 8) teilweise entbehrlich. Ja, die planmäßige Gestaltung der Fläche als Kohäsionsmittel entlastet Grammatik so sehr, dass sogar in längeren Texten (wie in unserem Beispiel Abb. 9) traditionelle Syntax teilweise aufgelöst wird. ${ }^{50}$

Tertiäre Schriftlichkeit in verdichteter, multimodaler Kommunikation verzichtet auf diejenigen Elemente, die typischerweise und spezifisch für primäre Schriftlichkeit in zerdehnter, monomodaler Kommunikation notwendig waren und sind. Es verhält sich wie immer in der Sprachgeschichte, nämlich „daß funktionslos gewordene Teile der Rede schwinden“ (Horn 1923, S. 2) ${ }^{51}$ Oder, etwas umständlicher, aber auch präziser formuliert: Um kommunikativen Aufwand und kommunikatives Ergebnis in ein möglichst effizientes Verhältnis zu bringen, bedarf es umso weniger ausgefeilter Grammatik, je massiver Ordnungssysteme nonverbaler Modi mitwirken. ${ }^{52}$

Im multimodalen Zusammenhang der Sehfläche Bildschirm tritt Grammatik einige ordnende Funktionen an Design ab, wird also schlanker. Wenn - mit Weinrich (1988, S. 86) - „die Grammatik in ihrem anthropologischen Kernbereich nichts anderes ist als das Gedächtnis des Leibes oder genauer gesagt zweier Leiber", so braucht man das bei leibentbundener, technisierter Kommunikation nicht mehr in dieser Weise und in diesem Maße. Denn Erinnerung wird in erheblichem Grade an leibferne Quellen delegiert ${ }^{53}$ die in multimodalem Zusammenspiel auf unterschiedlichste Ordnungsformen zurückgreifen.

50 Dieser linguistische Befund über profane Sehflächen modifiziert und schärft die allgemeine und teils metaphorisch formulierte Bemerkung von Weiss (1984, S. 16) über künstlerische Seh-Texte: „Die grammatikalische Syntax wird durch eine Bild-Syntax ergänzt bzw. ersetzt.“

51 Im Anschluss an einen Gedanken von Behaghel greift Horn mit dieser Auffassung die Lehre von der Allgemeingeltung der Lautgesetze an: „,Wenn es richtig ist, daß funktionslose Bestandteile der Rede schwinden, entgegen den ,Lautgesetzen', dann ist es auch sehr wohl denkbar, daß funktionswichtige Bestandteile bewahrt bleiben den ,Lautgesetzen“ zum Trotz. Die Funktion beherrscht das Lautgeset\%:" (Horn 1923, S. 131).

52 Vgl. den Überblick über „Elemente effizienter Kommunikation“ bei Roelcke (2002, S. 70), der für multimodale Kommunikate an einigen Stellen spezifiziert und ergänzt werden kann.

53 Vgl. schon Platon $(1958$, S. 55 = 275a) über Schrift: „Denn diese Erfindung wird den Seelen der Lernenden vielmehr Vergessenheit einflößen aus Vernachlässigung der Erinnerung““. 
Schrift in der Nähe von Bildern, vor allem auf dicht gefüllten Bildschirmflächen, neigt zu Kleinteiligkeit: einfache Module statt komplexer Strukturen, sichtbares Nebeneinander statt intellektuell zu erschließender Hierarchie, analytischer statt synthetischer Sprachbau. ${ }^{54}$

Das entspricht auch den Vorschlägen, die in Stilratgebern für Webdesigner gegeben werden, prägnant zusammengefasst in der Maxime „Keep it short or break it up!“汭 „Der Texter portioniert seine Information in für sich stehende und in sich verständliche Module. Er bricht den Text in Informationshäppchen auf (Break it up!), die der User anklicken kann, wenn er will (Detail on Demand). ${ }^{656}$

Text auf Webseiten liefert oft Kleinstinhalte (microcontent), z.B. in Titeln, Überschriften, Teasern, ${ }^{57}$ Index-Elementen, Bildlegenden und anderen Paratexten, webspezifisch aber auch in Orientierungshilfen, Linkbeschriftungen und sonstigen Navigations- und Aufmerksamkeitssteuerern. ${ }^{58}$ Die kleinteilig-modulare Architektur ohne größere Sinnbögen strahlt auch auf etwas längere Texte aus, wie sie seltener auf der ersten und häufiger auf der zweiten Ebene einer Website stehen.

Alles in allem wird das Verhältnis von Teilen und Ganzen in einem Zusammenspiel von Grammatik und Design organisiert: Grammatik und Design teilen sich die architektonische Aufgabe der Konstruktion von Sinn. Je augenfälliger die visuelle Gestaltung der Sehfläche Botschaften trägt und den Handlungsraum ihrer Nutzerin oder ihres Nutzers gliedert, ${ }^{59}$ desto weniger braucht Grammatik die verbalen Anteile zu strukturieren. Design entlastet Grammatik; Grammatik kann folglich einfacher (weniger komplex) werden.

In anderer Weise kennen wir ein solches Zusammenspiel zwischen Sprachgrammatik und Ordnungssystemen nonverbaler Modi bereits aus der unmittelbaren mündlichen Kommunikation. In dem Maße, wie die Ge-

54 Im menschheitsgeschichtlichen Maßstab argumentiert Wunderlich (2008) dafür, dass Morphologie „möglicherweise älter als Syntax“ (S. 254) und heute „unnütz“ (S. 252) sei. Reiche Morphologie sei „ein Übergangszustand von Sprache, die im Zuge der Bevölkerungszunahme zunächst um eine transparente Syntax ergänzt und später vielfach reduziert wurde“ (S. 258). In dieser Perspektive könnte, eine Epoche später, die Syntax von Schriftlichkeit in technisiert-multimodaler Umgebung zunächst um visuelles Design ergänzt und später, wo unnütz geworden, vielfach reduziert werden. Das würde auch universale Verständigung über die Grenzen einzelner Sprachen hinweg erleichtern. Vielleicht wären wir dann weniger auf dem Weg zu global Englishes als vielmehr zu global visual design.

55 Amy Gahran zugeschrieben, z.B. laut Alkan (2002, S. 28).

56 Alkan (2002, S. 29). Ähnlich z.B. Radtke/Pisani/Wolters (2006, S. 258 f.) u.v.a.

57 Teaser, im Web meist auf anklickbaren Teaserflächen, kündigen als Kurztexte jeweils einen längeren Text an.

58 „Microcontent is all the short bits of text that help guide the user or provide an ,at-a-glancec overview of what a given page is about." (Gahran 2002).

59 „Durch das Design des Interface wird der Handlungsraum des Nutzers von Produkten gegliedert." (Bonsiepe 1996, S. 26). 
sprächspartner auf Informationen aus dem gemeinsamen Wahrnehmungsraum zurückgreifen und sich mit Gestik, Mimik, Blickkontakt und direkter Körperkommunikation verständigen, kommt der dabei gesprochene Wortlaut mit knappen und entsprechend grammatikarmen Ellipsen aus. ${ }^{60} \mathrm{In}$ technisch vermittelter dichter Kommunikation wird das multimodale Setting mit Geräten hergestellt, aber auch hier ist Sprache mit anderen Modi verwoben. So wird Kress/Van Leeuwens (2001, S. 4) Gedanke plausibel, dass multimodale Texte im Gegensatz zu reiner Sprache nicht mit doppelter, sondern mit vielfacher Artikulation arbeiten: „,we see multimodal texts as making meaning in multiple articulations" ${ }^{\text {. }}{ }^{1}$

\section{Multimodale Ausdrucksformen}

\subsection{Articulirte Pixel (mit fünftem und sechstem Beispiel)}

Natürlich werden wir weiterhin die geniale Erfindung unserer Vorfahren nutzen, mittels ,doppelter Artikulation' (Martinet 1963, S. 21 f.) zu kommunizieren, also mit einer großen Anzahl bedeutungstragender Morpheme, die aus einer kleinen Menge bedeutungsunterscheidender Phoneme zusammengesetzt werden. Schon für primäre Schriftlichkeit in zerdehnter Kommunikation reicht das aber nicht aus: Spatien, Interpunktion und andere visuelle Lesehilfen kommen hinzu. Tertiäre Schriftlichkeit in verdichteter Kommunikation nutzt, wie wir sahen, weitere Mittel zur visuellen Artikulation von Botschaften. Pixel auf Bildschirmen bieten dafür eine ideale materielle Grundlage.

So wie - nach Humboldt (1994d, S. 133) - ,alles Sprechen darin besteht, die schweifende Unbestimmtheit des blossen Vorstellens durch die Schärfe des articulirten Lautes zu heften“, so muss in der multimodalen Umgebung des World Wide Web „die schweifende Unbestimmtheit des blossen Vorstellens “ an ,die Schärfe articulirter Pixel' gebunden werden, um kommunizierbare Klarheit zu gewinnen. Die andere materielle Grundlage des Zeichens (Pixel statt Laut) erlaubt und erzwingt andere Wege der Artikulation, also der Form(ulier)ung von Vorstellungen. Eine visuell durchgestaltete Sehfläche aus dem Web zieht andere Arten von Botschaften und anderen Umgang damit nach sich.

60 Vgl. z.B. Bühler (1934, S. 155-158).

61 Vgl. ebd., S. 111 f. - Kress/Van Leeuwen (2001) beziehen ,Design' nicht auf die visuelle Gestaltung von Objekten, sondern auf die Gestaltung des multimodalen Zusammenspiels von Botschaften. „Design stands midway between content and expression. It is the conceptual side of expression, and the expression side of conception.“ (ebd., S. 5). ,[The idea of design is] a deliberateness about choosing the modes for representation, and the framing for that representation.“ (ebd., S. 45). „The design process reshapes, transforms, both writing and image, both as realisational material and as discourses (as well as scripts and genre), and as existing potential shapings." (ebd., S. 64). 


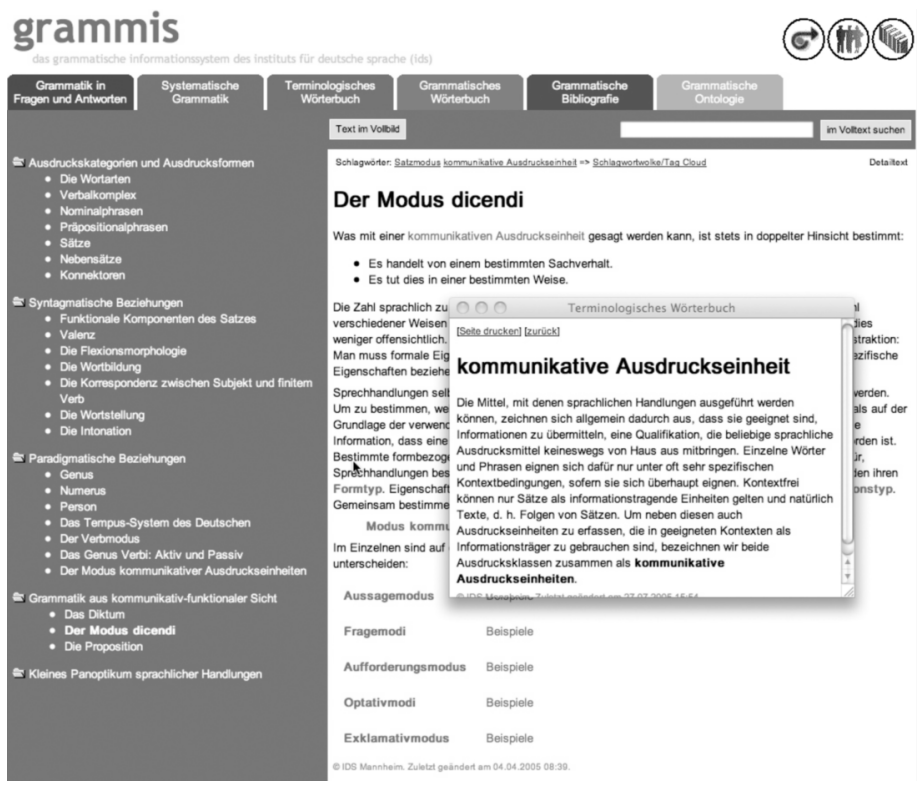

Abb. 10: Grammis ${ }^{62}$

\section{KIA ERHÖHT DIE UMWELTPRÄMIE!}
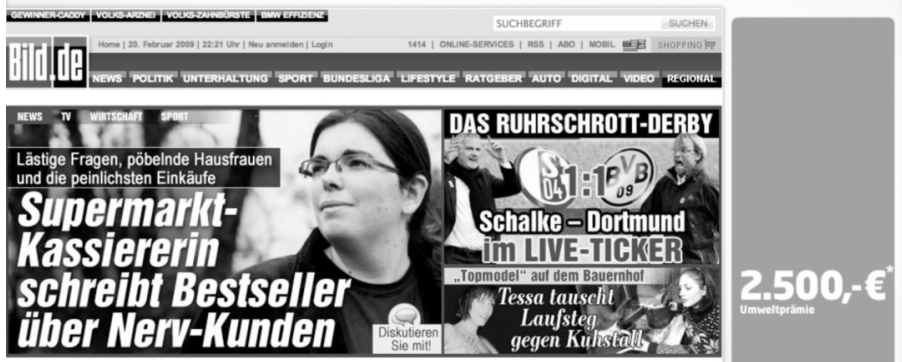

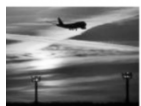

neues RANking

Fliegen am

sichersten?

Ein nouvos Ranking dor IATA
20igt, wo Fliogon am

sichersiten ist und wo

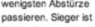

(asien. mont
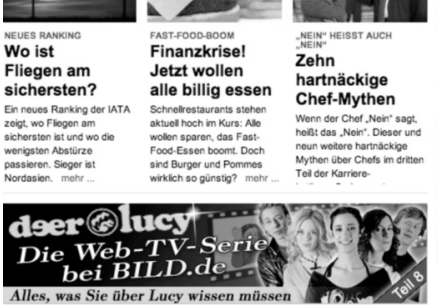
Finanzkrise Jetzt wollen alle billig esse Schnolvestaurants stoher (t) ood-Essen boomt Doch
NEIN" HEISST AUOA Zehn

hartnäckige Chef-Mythen Wenn dor Chet ,Nein' sagt, (a) Tei dee Karriere.

niswsichen

21:23 UHR Oberstes Schwoizer Gericht verbiolo

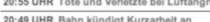
20:49 UHR Bahn kündigt Kurzarboil an 20:44 UHR Rötgens mit USA-Rolso zutrieden

Abb. 11: bild.de (www.bild.de (Stand: 20.2.2009)

62 http://hypermedia.ids-mannheim.de/pls/public/sysgram.ansicht?v_typ=d\&v_id=2044 (Stand: 20.2.2009), 
Grammis, das grammatische Informationssystem des IDS, bildet nicht geradewegs die in Buchform erschienene IDS-Grammatik (Zifonun et al. 1997) ab; bild.de ist nicht die für den Bildschirm übersetzte Bild-Zeitung. ${ }^{63}$ Beispielsweise nutzt Grammis im Druck unmögliche Hyperlinks mit der Folge anderer Textauswahl und -aufteilung; und die hier gezeigte Titelseite von bild.de enthält viel mehr und andere, meist kleinflächigere Einheiten und kürzere Texte als die gedruckte Titelseite des gleichen Tages. Systematische Untersuchungen charakteristischer Merkmale unterschiedlicher Text-BildSorten in verschiedenen Kommunikationsformen gibt es noch nicht. Jeder Nutzer und jede Verfasserin hat aber - wie beim kindlichen Spracherwerb mindestens ein intuitives Wissen über unterschiedliche Leistungen, Möglichkeiten und Qualitäten gesprochener, geschriebener, multimodaler und hypermedialer Texte erlangt und kann mehr oder weniger geschickt damit umgehen.

Ist Sprache - wiederum mit Humboldt (1963, S. 418) ${ }^{64}$ - „die sich ewig wiederholende Arbeit des Geistes, den articulirten Laut zum Ausdruck des Gedanken fähig zu machen", so arbeitet der Webseitengestalter mit multimodalen Ressourcen, um articulirte Pixel (und ggf. Töne etc.) ${ }^{65}$ ausdrucksfähig zu machen. Während „die Verwandlung der Welt in Sprache [...] auch vermittelst ihres grammatischen Baues" geschieht (Humboldt 1994e, S. 167), ${ }^{66}$ tritt auf Sehflächen Design als symbolische Kraft hinzu. Hatte Humboldt die „,intellectuellen Verfahren“ der Sprachen vergleichend untersucht, ${ }^{67}$ so stehen eine ähnlich vergleichende Design- und gar Multibzw. Hypermodalitätswissenschaft noch aus. Humboldt als Idealist konzentriert sich auf die Form des Ausdrucks, nicht die materielle Grundlage, lässt aber - vor allem im Vergleich von Lautsprache und Schriftsprache doch durchblicken, dass auch Stoff und Modus eine Rolle spielen. Schließlich liege „das ganze grammatische Streben der Sprache“ „in der Darstellung der Verstandeshandlung durch den Laut" (Humboldt 1994b, S. 58 f.). „Allein das tönende Wort ist gleichsam eine Verkörperung des Gedanken, die Schrift eine des Tons“ (Humboldt 1994c, S. 100); ,und so kann die Uebertragung des erst als Ton gehefteten Gedanken auf einen Gegenstand des Auges, nach Massgabe der Art, wie sie geschieht, dem Geiste sehr ver-

63 Zumal das Hauptfeld oben links sich alle paar Sekunden verändert und ein Klick auf das Foto unten rechts zu einem Videofilm führt.

64 Eine zusammenfassende Darstellung des artikulatorischen Geschäfts der Sprache nach Humboldt gibt Trabant (2008, S. 25-46).

65 Wie gesagt: Dieser Beitrag konzentriert sich auf Schrift an Bild. Das sind nur zwei Fäden aus in vielen Fällen tatsächlich komplexerer Multimodalität (mit gesprochener Sprache, Ton, Animation u.a. sowie ggf. Bedingungen der Rezeptionssituation).

66 Vgl. „die sprachbildende Kraft der Sprache in dem Acte der Verwandlung der Welt in Gedanken“ (Humboldt 1963, S. 413).

67 „Es kann scheinen, als müssten alle Sprachen in ihrem intellectuellen Verfahren einander gleich seyn." (Humboldt 1963, S. 464). 
schiedne Richtungen geben. Offenbar aber müssen, wenn die Gesammtwirkung nicht gestört werden soll, das Denken in Sprache, die Rede und die Schrift übereinstimmend gebildet, und wie aus Einer Form gegossen seyn.“ (Humboldt 1994c, S. 100).

Das hier angestrebte Ideal der gleichen Grammatik für Laut- und Schriftsprache kann, soweit überhaupt, natürlich nur dort gelten, wo ein Modus (geschriebene Sprache) den anderen (gesprochene Sprache) ersetzen soll (partiell also in primärer Schriftlichkeit). In bi- oder multimodalen Konstellationen wie auf Sehflächen hingegen ergänzen verschiedene Modi einander. Gerade aus dem synergetischen Zusammenspiel von Design und Grammatik und damit eben auch ,verschiedner Richtungen des Geistes“ soll sich eine „Gesammtwirkung“ ergeben, die im Idealfall Teilleistungen einzelner Modi übersteigt. Die Architektur doppelter Artikulation von Sprache wird eingebaut in die multiple Artikulation multimodaler Diskurse.

\subsection{Schweifende Unbestimmtheit}

Unterschiedliche materielle Zeichenträger ziehen also unterschiedliche Ausdrucksformen nach sich und können andere Denkweisen nahelegen. Ein Bild kann nicht ohne weiteres in einen Text übersetzt werden, ein kohärenter Ganztext nicht umstandslos in einen Hypertext, primäre nicht einfach in tertiäre Schriftlichkeit und jeweils umgekehrt. Konkret lässt sich das nur an einzelnen Fällen studieren; und kein Material setzt der „Unbestimmtheit des blossen Vorstellens" (Humboldt 1994d, S. 133) unverrückbare Grenzen. Jedes Material aber eignet sich für unterschiedliche Zwecke unterschiedlich gut und fordert andere Gestaltungskräfte heraus, erst recht in Verbindung mit neuen Medien und Kommunikationsformen.

So wirkt Koch/Oesterreichers (1985 und 1994) damals wegweisende Unterscheidung in mediale bzw. konzeptionelle Mündlichkeit und Schriftlichkeit mittlerweile etwas altmodisch - und zwar nicht nur, wie Dürscheid (2003) zeigt, wegen eines zu engen Medienbegriffs und neuer (quasi-synchroner) Kommunikationsformen, sondern weil diese beiden sprachlichen sehr oft von nonverbalen Modi begleitet und beeinflusst werden. Wir werden über konzeptionelle Bildlichkeit ${ }^{68}$ und konzeptionelle Bi- und Multimodalität nachdenken müssen. Der Alltagsmensch beherrscht sie längst. Möglicherweise entstehen auch neue Weisen der Weltaneignung (Cassirer 1923-1929) bzw. Welterzeugung (Goodman 1984) daraus. Das kann an dieser Stelle nur angedeutet werden und ist eingehende Untersuchungen wert.

Kress/Van Leeuwen (2001, S. vii) weisen zu Recht darauf hin, dass die Frage, was ein Modus ist, viel weniger fruchtbar ist als die Frage, wie Menschen die Vielfalt semiotischer Ressourcen nutzen, um in konkreten gesellschaftlichen Zusammenhängen Zeichen zu erzeugen. Tatsächlich gebe es 68 Allgemein einführend zu Bildern überhaupt Bruhn (2009). 
weder eine strikte Arbeitsteilung noch undurchlässige Grenzen zwischen verschiedenen Kommunikationsmodi (z.B. Bild, Ton, Musik im Film) (ebd. S. 2, 125). Vielmehr, so können wir sagen, nutzen Menschen sie in unterschiedlicher Mischung je nach den gegebenen Zwecken und Möglichkeiten.

Relevante Unterschiede zwischen Modi liegen, so Kress/Van Leeuwen (2001, S. 126), nicht in physischen Beschränkungen des verwendeten Materials, sondern in dem Maß an Arbeit, das eine Gesellschaft hineingesteckt hat, um es im Rahmen multimodaler Ensembles für kommunikative Zwecke tauglich zu machen - oder, wie Humboldt sagen würde, um es zum Ausdruck des Gedanken fähig zu machen. In manchen Konstellationen, das können wir beobachten, werden z.B. Gebärden für besonders geeignete semiotische Ressourcen gehalten, in anderen Laute, Lettern oder eben Pixel.

Tatsächlich aber ist multimodale Kommunikation der Normalfall..$^{69}$ Das war durch die lange Vorrangstellung monomodal schriftlicher Text im Zeitalter des Buchdrucks (also wegen technischer Beschränkungen in zerdehnter Kommunikation) aus dem Blick geraten. „In every mode of the multimodal ensemble there is always 'work' with all the available representational forms, and such work is always meaningful." (Kress/Van Leeuwen 2001, S. 112).

Ich habe mich hier auf die Veränderung von Schrift im Konzert multimodaler Kommunikation konzentriert. Ein vollständiges Bild zeigt sich erst, wenn das Zusammenspiel aller semiotischer Ressourcen im Hinblick auf die jeweiligen Verständigungszwecke betrachtet wird. Alltagsmenschen tun das immer schon mehr oder weniger spontan. Die Arbeit wissenschaftlicher Empirie und Reflexion kann helfen, transparenter, verständlicher, verfügbarer und vielleicht auch besser zu machen, was wir tun.

\section{Ausblick}

\subsection{Performative Effizienz}

Formen der Organisation und Übermittlung von Sinn richten sich danach, was sich für erfolgreiche Kommunikation unter den gegebenen Konstellationen als am effizientesten erweist. Das führt in (mündlich-multimodaler) unmittelbarer, (schriftlich-monomodaler) zerdehnter und (technisiert-multimodaler) verdichteter Kommunikation zu jeweils anderen Formaten und Figuren. So schlagen materielle Grundlagen, bei unserem Thema das technische Arrangement von Pixeln auf Bildschirmen zum hypermedialen World Wide Web, auf die Formung der Sprache durch. Die - in Humboldts Diktion „sich ewig wiederholende Arbeit des Geistes“, articulirte Pixel zum Aus-

$69 \quad,[\mathrm{M}]$ eaning is made in many different ways, always, in the many different modes and media which are co-present in a communicational ensemble.“ (Kress/Van Leeuwen 2001, S. 111). 
druck von Botschaften fähig zu machen, erzeugt neuartige Text-Bild-Gestalten, in denen Grammatik und Design als Institutionen der Ordnung zusammenspielen, welche sich in ihrem Vollzug ständig erneuern.

Autonome (monomodale) Schriftlichkeit musste vom Ich/Du-JetztHier der unmittelbaren Face-to-Face-Kommunikation abstrahieren, um zerdebnte Kommunikation überhaupt möglich zu machen. Verdichtete Kommunikation heute holt, wie Clooneys Eingangsbeispiel (Kap. 1.1) zeigte, mit digitalen Techniken immer mehr Elemente von Situiertheit und Verkörperung zurück in kommunikative Ereignisse, wenngleich in technisch konstruierter Weise. So wirkt sie lebendiger, schneller, vielseitiger, beweglicher, performativer als rein schriftlicher Austausch ${ }^{70}$ und kann deshalb auch tiefer in unmittelbare Kommunikation eingebettet werden. Schnelle Wechsel, handelnde Teilhabe, transparente Inszenierung, aktuelle Aufführung und semiotische Kreativität kommen in multimodalem Verkehr - wenngleich technisch vermittelt - spürbarer und körpernäher zur Geltung als in rein schriftlichen Texten. ${ }^{71}$ Der schriftaffine ideologische Unterschied von res cogitans und res extensa wird unterminiert.

All das gilt umso stärker, je beweglicher Sehflächen sind, im Web also je mehr Hyperlinks genutzt werden. Doch auf Hyperformative konnte ich im beschränkten Rahmen dieses Aufsatzes nicht eingehen.

Wie Gesellschaften insgesamt werden mit der Zeit auch ihre Kommunikationsinstrumente komplexer - zumal die alten neben den neuen und teils unter ihrem Einfluss ja fortbestehen. Tertiäre Schriftlichkeit verdrängt nicht die alten Formen, sondern ergänzt und erweitert sie, eröffnet also neue Potentiale, die - wie immer - unterschiedlich intensiv und unterschiedlich gut genutzt werden können. Denken wir an den antiken Schreiber, der beim Wechsel von Mündlichkeit zu Schriftlichkeit das Trägermaterial ${ }^{72}$ tauscht: „die flüchtige Luft gegen den beständigen Papyrus, eine zeitliche gegen eine räumliche Ordnung, Sukzession gegen Linearität. Die Frage ist, ob er die Möglichkeiten, die das neue Medium bietet, genutzt hat und - inwieweit. Er hat von ihnen“, so meint Ludwig (2005, S. 60), „nur in einem sehr bescheidenen Maße Gebrauch gemacht".

$70 \quad$ Nicht zufällig erlebte die wissenschaftliche Isolierung sprachlicher Kompetenz von zugleich abgewerteter Performanz ihren Höhepunkt am Ende des Gutenberg-Zeitalters, als die ersten Computer mit Schrift programmiert werden konnten.

71 Vgl. Wulf/Zirfas (2005, S. 8): „Die Perspektive des Performativen rückt die Inszenierungsund Aufführungspraktiken sozialen Handelns, deren wirklichkeitskonstitutive Prozesse sowie den Zusammenhang von körperlichem und sprachlichem Handeln, Macht und Kreativität in den Mittelpunkt" und ,führt zu einem performativen Wahrnehmungs- und Raumbegriff, in dessen Rahmen die Rhythmen des Zusammenspiels von Ordnung und Bewegung, Erinnerung und Erwartung, Teilhabe und Distanz neu in den Blick geraten" (ebd., S. 12). - Zur aktuellen Debatte über Medialität und Performanz vgl. etwa Krämer (2000), Krämer (Hg.) (2004), Schneider (2008).

72 Ludwig (2005, S. 60) schreibt hier „Medium“. 
Wer sich im Web artikuliert, tauscht bewegliche Lettern gegen einzeln programmierbare Pixel, langsames, fest bedrucktes Papier gegen schnelle, veränderliche Bildschirme, materiell räumliche Ordnung gegen zwar virtuell räumliche, oberflächlich sichtbar jedoch flächige und zeitlich bewegliche Ordnung, bedächtiges Lesen gegen flottes Streunen, kurz: überschaubar stabile Informationswelten gegen unendlich variable Komplexität. Einige Beispiele haben wir oben betrachtet. Es ist aber wahrscheinlich, dass es nicht - wie bei Schrift - Jahrtausende dauern wird, bis die kommunikativen Möglichkeiten, welche die neuen materiellen Eigenschaften und technischen Bedingungen bieten, auch erkundet und ausgenutzt werden, sondern nur Jahrzehnte.

\subsection{Siebtes Beispiel: Bestimmt ausschweifen}

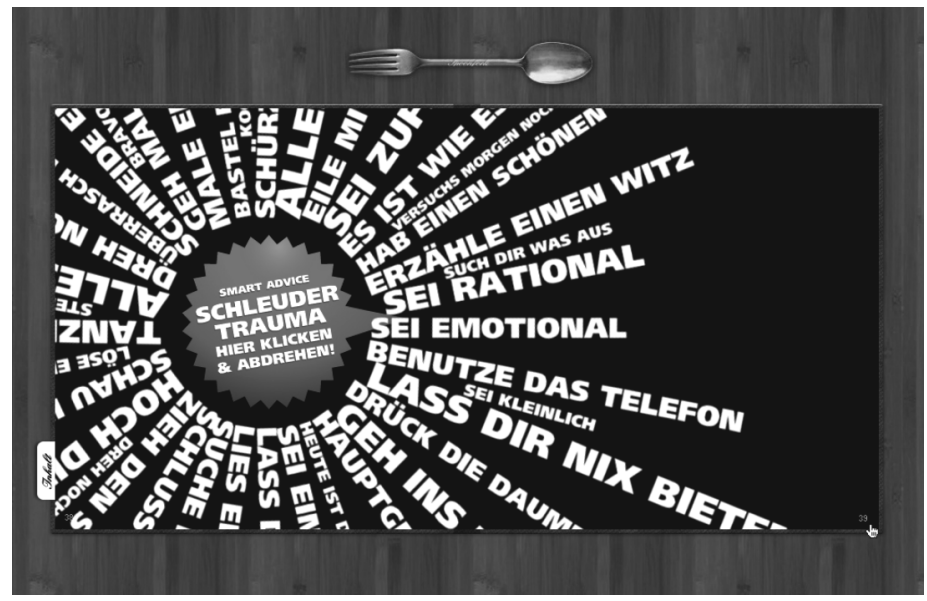

Abb. 12: www.spoonfork.de (Stand: 9.2.2009)

Abb. 12 kann, weil in die primäre Schriftlichkeit dieses Aufsatzes gezwängt, nur eine magere schwarzweiße Reproduktion eines bewegungslos gestellten Ausschnittes vergleichsweise weit entwickelter tertiärer Schriftlichkeit geben. Der geneigte Leser möge deshalb jetzt umschalten zu www.spoonfork.de und dort frei umherschweifen. In diesem Online-Magazin wird (jeden Monat neu) ein präzise arrangiertes Feuerwerk webgestalterischer Möglichkeiten vorgeführt. Hier tanzen Pixel, beleben den Bildschirm, erfrischen Schrift an Bild. Eine multimodale Vielfalt technisch-semiotischer Ressourcen wird in einer Weise durchgearbeitet, welche die schweifende Unbestimmtheit des Vorstellens teils in bewegliche Bahnen lenkt, teils noch weiter ausschweifen lässt. Humboldt hätte seine Freude daran. 


\section{Literatur}

Alkan, Saim Rolf (2002): Texten für das Internet. Ein Handbuch für Online-Redakteure und Webdesigner. Bonn.

Bonsiepe, Gui (1996): Interface. Design neu begreifen. Mannheim.

Brecht, Bertolt (1967): Turandot oder Der Kongreß der Weißwäscher. In: Brecht, Bertold: Gesammelte Werke in 20 Bänden. Bd. 5. Frankfurt a.M., S. 2193-2270.

Brinker, Klaus (2001 [1985]): Linguistische Textanalyse. Eine Einführung in Grundbegriffe und Methoden. 5. Aufl. Berlin.

Bruhn, Matthias (2009): Das Bild. Theorie - Geschichte - Praxis. Berlin.

Bühler, Karl (1934): Sprachtheorie. Die Darstellungsfunktion der Sprache. Jena.

Canetti, Elias (1936): Die Blendung. Roman. Wien/Leipzig/Zürich.

Cassirer, Ernst (1923-1929): Philosophie der symbolischen Formen. 3 Bde. Berlin.

Costall, Alan (2007): Bringing the body back to life: James Gibson's ecology of agency. In: Ziemke, Tom/Zlatev, Jordan/Frank, Roslyn M. (Hg.): Body, language and mind. Bd. 1: Embodiment. Berlin/New York, S. 55-83.

Detel, Wolfgang/Schramm, Matthias/Borsche, Tilman/Piepmeier, Rainer/Hucklenbroich, Peter (1980): [Artikel] Materie. In: Ritter/Gründer/Gabriel (Hg.) (19712007), Bd. 5., Sp. 870-924.

Dürscheid, Christa (2003): Medienkommunikation im Kontinuum von Mündlichkeit und Schriftlichkeit. Theoretische und empirische Probleme. In: Zeitschrift für Angewandte Linguistik 38, S. 37-56.

Eckkrammer, Eva Martha/Held, Gudrun (Hg.) (2006): Textsemiotik. Studien zu multimodalen Texten. Frankfurt a.M. u.a.

Ehlich, Konrad (1983): Text und sprachliches Handeln. Die Entstehung von Texten aus dem Bedürfnis nach Überlieferung. In: Assmann, Aleida/Assmann, Jan/Hardmeier, Christof (Hg.): Schrift und Gedächtnis. Beiträge zur Archäologie der literarischen Kommunikation. München, S. 24-43.

Engels, Friedrich (1962 [1876]): Dialektik der Natur. Anteil der Arbeit an der Menschwerdung des Affen. In: Marx, Karl/Engels, Friedrich: Werke. Bd. 20. Berlin, S. $444-455$.

Ernst, Ulrich (2003): Die Kreuzgedichte des Hrabanus Maurus als multimediales Kunstwerk. Textualität - Ikonizität - Numeralität. In: Schmitz, Ulrich/Wenzel, Horst (Hg.): Wissen und neue Medien. Bilder und Zeichen von 800 bis 2000. Berlin, S. 13-37.

Fischer, Martin (1997): Schrift als Notation. In: Koch/Krämer (Hg.), S. 83-101.

Freyermuth, Gundolf S. (2005): Der große Kommunikator. Soziale Konsequenzen von media merging und Transmedialisierung. In: Siever/Schlobinski/Runkehl (Hg.), S. $15-45$.

Füssel, Stephan (1999): Gutenberg und seine Wirkung. Frankfurt aM./Leipzig. 
Gahran, Amy (2002): In online writing, little things mean a lot. www.contentious.com/ 2006/03/14/in-online-writing-little-things-mean-a-lot (Stand: Januar 2009).

Genette, Gérard (1989): Paratexte. Das Buch vom Beiwerk des Buches. Frankfurt a.M. [frz. 1987].

Gibbs, Raymond W. (2006): Embodiment and cognitive science. Cambridge u.a.

Giesecke, Michael (2006 [1991]): Der Buchdruck in der frühen Neuzeit. Eine historische Fallstudie über die Durchsetzung neuer Informations- und Kommunikationstechnologien. 4. Aufl. Frankfurt a.M. [1. Aufl. Frankfurt a.M. 1991].

Goodman, Nelson (1984): Weisen der Welterzeugung. Frankfurt a.M. [amerik. 1978].

Greber, Erika/Ehlich, Konrad/Müller, Jan-Dirk (Hg.) (2002): Materialität und Medialität von Schrift. Bielefeld.

Gumbrecht, Hans Ulrich/Pfeiffer, K. Ludwig (Hg.) (1988): Materialität der Kommunikation. Frankfurt a.M.

Günther, Hartmut/Ludwig, Otto (Hg.) (1994): Schrift und Schriftlichkeit. Ein interdisziplinäres Handbuch internationaler Forschung. 1. Halbbd. (= Handbücher zur Sprach- und Kommunikationswissenschaft 10.1). Berlin/New York.

Habscheid, Stephan (2000): ,Medium“ in der Pragmatik. Eine kritische Bestandsaufnahme. In: Deutsche Sprache 28, S. 126-143.

Harris, Roy (2008): Die integrale Zeichenkonzeption. In: Zeitschrift für Semiotik 30, S. $11-28$.

Hölderlin, Friedrich (1975-2008): Sämtliche Werke. Hrsg. v. Dietrich E. Sattler. Frankfurt a.M./Basel u.a. [,Frankfurter Ausgabe"].

Hörisch, Jochen (1992): Brot und Wein. Die Poesie des Abendmahls. Frankfurt a.M.

Hoffmann, Stefan (2002): Geschichte des Medienbegriffs. (= Archiv für Begriffsgeschichte, Sonderheft 2002). Hamburg.

Horn, Wilhelm (1923 [1921]): Sprachkörper und Sprachfunktion. 2. Aufl. Leipzig.

Humboldt, Wilhelm von (1963 [1830-1835]): Ueber die Verschiedenheit des menschlichen Sprachbaues und ihren Einfluss auf die geistige Entwicklung des Menschengeschlechts [= Einleitung zum Kawiwerk]. In: Humboldt, Wilhelm von: Werke in fünf Bänden. Hrsg. v. Andreas Flitner und Klaus Giel. Bd. III: Schriften zur Sprachphilosophie. Darmstadt, S. 368-756. [Originalausg. 1830-1835].

Humboldt, Wilhelm von (1994a): Über die Sprache. Reden vor der Akademie. Hrsg. v. Jürgen Trabant. Tübingen/Basel.

Humboldt, Wilhelm von (1994b [1822]): Ueber das Entstehen der grammatischen Formen, und ihren Einfluss auf die Ideenentwicklung. In: Humboldt (1994a), S. 52 81. [Originalausg. 1822].

Humboldt, Wilhelm von (1994c [1824]): Ueber die Buchstabenschrift und ihren Zusammenhang mit dem Sprachbau. In: Humboldt (1994a), S. 98-125. [Originalausg. 1824].

Humboldt, Wilhelm von (1994d [1826]): Ueber den grammatischen Bau der Chinesischen Sprache. In: Humboldt (1994a), S. 126-142. [Originalausg. 1826]. 
Humboldt, Wilhelm von (1994e [1827]): Ueber den Dualis. In: Humboldt (1994a), S. 143-169. [Originalausg. 1827].

Ingenhoff, Diana/Schmitz, H. Walter (2000): Über den Gegenstand gesprächsanalytischer Transkriptionen. In: Hess-Lüttich, Ernest W. B./Schmitz, H. Walter (Hg.): Botschaften verstehen. Kommunikationstheorie und Zeichenpraxis. Festschrift für Helmut Richter. Frankfurt a.M. u.a., S. 143-154.

Jäger, Ludwig (2008): Aposème und Parasème: Das Spiel der Zeichen - Saussures semiologische Skizzen in den „Notes“. In: Zeitschrift für Semiotik 30, S. 49-71.

Koch, Peter/Krämer, Sybille (Hg.) (1997): Schrift, Medien, Kognition. Über die Exteriorität des Geistes. Tübingen.

Koch, Peter/Oesterreicher, Wulf (1985): Sprache der Nähe - Sprache der Distanz. Mündlichkeit und Schriftlichkeit im Spannungsfeld von Sprachtheorie und Sprachgeschichte. In: Romanistisches Jahrbuch 36, S. 15-43.

Koch, Peter/Oesterreicher, Wulf (1994): Schriftlichkeit und Sprache. In: Günther/Ludwig (Hg.), S. 587-604.

Krämer, Sybille (1997): Schrift und Episteme am Beispiel Descartes'. In: Koch/Krämer (Hg.), S. 105-126.

Krämer, Sybille (2000): ,Performativität' und ,Verkörperung‘. Über zwei Leitideen für eine Reflexion der Medien. In: Pias, Claus (Hg.): Neue Vorträge zur Medienkultur. Weimar, S. 185-201.

Krämer, Sybille (2003): > Schriftbildlichkeit< oder: Über eine (fast) vergessene Dimension der Schrift. In: Krämer, Sybille/Bredekamp, Horst (Hg.): Bild - Schrift - Zahl. München, S. 157-176.

Krämer, Sybille (Hg.) (2004): Performativität und Medialität. München.

Krämer, Sybille (2008): Medium, Bote, Übertragung. Kleine Metaphysik der Medialität. Frankfurt a.M.

Kress, Gunther (2003): Literacy in the New Media Age. London/New York.

Kress, Gunther/Van Leeuwen, Theo (2001): Multimodal discourse. The modes and media of contemporary communication. London.

Kroeber-Riel, Werner (1993): Bildkommunikation. Imagerystrategien für die Werbung. München.

Leroi-Gourhan, André (1980): Hand und Wort. Die Evolution von Technik, Sprache und Kunst. Frankfurt a.M. [frz. 1964/1965].

Linck, Dirck/Rentsch, Stefanie (Hg.) (2007): Bildtext - Textbild. Probleme der Rede über Text-Bild-Hybride. Freiburg i.Br. u.a.

Ludwig, Otto (2005): Geschichte des Schreibens. Bd. 1: Von der Antike zum Buchdruck. Berlin/New York.

Luther, Martin (1521): Assertio omnium articulorum M. Lutheri per bullam Leonis X. novissimam damnatorum. In: Luther, Martin (1883 ff.): D. Martin Luthers Werke. Kritische Gesammtausgabe. Bd. 7. Weimar, Sp. 91-151. 
Malinowski, Bronislaw (1974): Das Problem der Bedeutung in primitiven Sprachen. In: Ogden, Charles K./Richards, Ivor Armstrong (Hg.): Die Bedeutung der Bedeutung. Eine Untersuchung über den Einfluß der Sprache auf das Denken und über die Wissenschaft des Symbolismus. Frankfurt a.M., S. 323-384. [engl. 1923].

Martinet, André (1963): Grundzüge der Allgemeinen Sprachwissenschaft. Stuttgart u.a. [frz. 1960].

Meier, Stefan (2008): (Bild-)Diskurs im Netz. Konzept und Methode für eine semiotische Diskursanalyse im World Wide Web. Köln.

Mon, Franz (1995 [1959]): artikulationen. In: Mon, Franz: Gesammelte Texte 2: Poetische Texte 1951-1970. Berlin, S. 5-39. [hier: „text und lektüre“, S. 9 f.].

Ong, Walter J. (1987): Oralität und Literalität. Die Technologisierung des Wortes. Opladen. [amerik. 1982].

Peirce, Charles Sanders (1967): Einige Konsequenzen aus vier Unvermögen [amerik. 1868]. In: Peirce, Charles Sanders: Schriften zum Pragmatismus und Pragmatizismus. Hrsg. v. Karl-Otto Apel. Frankfurt a.M., S. 40-87.

Pfeiffer, K. Ludwig (1988): Materialität der Kommunikation? In: Gumbrecht/Pfeiffer (Hg.), S. 15-28.

Piaget, Jean (1975): Nachahmung, Spiel und Traum. Die Entwicklung der Symbolfunktion beim Kinde. Stuttgart. [frz. 1945].

Platon (1958): Phaidros [griech. ca. 450 v. Chr.]. In: Platon: Sämtliche Werke. Hrsg. v. Walter F. Otto, Ernesto Grassi und Gert Plamböck. Bd. 4. Hamburg, S. 7-60.

Raab, Jürgen (2008): Visuelle Wissenssoziologie. Theoretische Konzeption und materiale Analysen. Konstanz.

Radtke, Susanne P./Pisani, Patricia/Wolters, Walburga (2006 [2001]): Handbuch Visuelle Mediengestaltung. 3. Aufl. Berlin.

Raible, Wolfgang (1991): Die Semiotik der Textgestalt. Erscheinungsformen und Folgen eines kulturellen Evolutionsprozesses. Heidelberg.

Ritter, Joachim/Gründer, Karlfried/Gabriel, Gottfried (Hg.) (1971-2007): Historisches Wörterbuch der Philosophie. 13 Bde. Basel.

Runkehl, Jens (2005): Text-Bild-Konstellationen. In: Siever/Schlobinski/Runkehl (Hg.), S. 202-218.

Roelcke, Thorsten (2002): Kommunikative Effizienz. Eine Modellskizze. Heidelberg.

Saussure, Ferdinand de (1967): Grundfragen der allgemeinen Sprachwissenschaft. Hrsg. v. Charles Bally und Albert Sechehaye. 2. dt. Aufl. Berlin. [frz. 1916].

Schmitz, Ulrich (2003): Lesebilder im Internet. Neue Koalitionen und Metamorphosen zwischen Text und Bild. In: Zeitschrift für Germanistik, Neue Folge XIII, 3/2003, S. $605-628$.

Schmitz, Ulrich (2006a): Tertiäre Schriftlichkeit. Text-Bild-Beziehungen im World Wide Web. In: Schlobinski, Peter (Hg.): Von *hdl* bis *cul8r*. Sprache und Kommunikation in den Neuen Medien. (= Thema Deutsch 7). Mannheim u.a., S. 89-103. 
Schmitz, Ulrich (2006b): Schriftbildschirme. Tertiäre Schriftlichkeit im World Wide Web. In: Androutsopoulos, Jannis K./Runkehl, Jens/Schlobinski, Peter/Siever, Torsten (Hg.): Neuere Entwicklungen in der linguistischen Internetforschung. (= Germanistische Linguistik 186-187). Hildesheim u.a., S. 184-208.

Schmitz, Ulrich (i.Dr.): Sehflächenforschung. Eine Einführung. In: Diekmannshenke, Hajo/Klemm, Michael/Stöckl, Hartmut (Hg.): Bildlinguistik. Berlin.

Schneider, Jan Georg (2006): Gibt es nichtmediale Kommunikation? In: Zeitschrift für Angewandte Linguistik 44, S. 71-90.

Schneider, Jan Georg (2008): Spielräume der Medialität. Linguistische Gegenstandskonstitution aus medientheoretischer und pragmatischer Perspektive. Berlin/New York.

Siever, Torsten (2005): Internetwerbung: Alter Wein in neuen Schläuchen? In: Siever/ Schlobinski/Runkehl (Hg.), S. 219-241.

Siever, Torsten/Schlobinski, Peter/Runkehl, Jens (Hg.) (2005): Websprache.net. Sprache und Kommunikation im Internet. Berlin/New York, S. 219-241.

Spitzmüller, Jürgen (2006 [2002]): Typographie. In: Dürscheid, Christa (Hg.): Einführung in die Schriftlinguistik. 3. Aufl. Göttingen, S. 207-238.

Stöckl, Hartmut (2004): Die Sprache im Bild - das Bild in der Sprache. Zur Verknüpfung von Sprache und Bild im massenmedialen Text: Konzepte. Theorien. Analysemethoden. Berlin/New York.

Storrer, Angelika (2001): Getippte Gespräche oder dialogische Texte? Zur kommunikationstheoretischen Einordnung der Chat-Kommunikation. In: Lehr, Andrea/ Kammerer, Matthias/Konerding, Klaus-Peter/Storrer, Angelika et al. (Hg.): Sprache im Alltag. Beiträge zu neuen Perspektiven in der Linguistik. Herbert Ernst Wiegand zum 65. Geburtstag gewidmet. Berlin/New York, S. 439-465.

Storrer, Angelika (2004): Text-Bild-Bezüge und Nutzermetaphern im World Wide Web. In: Mitteilungen des Deutschen Germanistenverbandes 51.1, S. 40-57.

Thaler, Verena (2005): Zur Problematik der Synchronizität computervermittelter Kommunikation. In: Zeitschrift für Angewandte Linguistik 43, S. 79-101.

Trabant, Jürgen (2008): Was ist Sprache? München.

Vlasic, Andreas/Schweiger, Wolfgang (1998): Bilder im World Wide Web. Ein Experiment zum Einfluß der Bebilderung in Online-Nachrichtenangeboten auf die Rezeption. In: Prommer, Elizabeth/Vowe, Gerhard (Hg.): Computervermittelte Kommunikation. Öffentlichkeit im Wandel. Konstanz, S. 39-65.

Wehde, Susanne (2000): Typographische Kultur. Eine zeichentheoretische und kulturgeschichtliche Studie zur Typographie und ihrer Entwicklung. Tübingen.

Weingart, Brigitte (2007): „Sehtextkommentar“. Zu den Bilderschriften und Schriftbildern Ferdinand Kriwets. In: Linck/Rentsch (Hg.), S. 85-116.

Weinrich, Harald (1988): Über Sprache, Leib und Gedächtnis. In: Gumbrecht/Pfeiffer (Hg.), S. 80-93. 
Weiss, Christina (1984): Seh-Texte. Zur Erweiterung des Textbegriffes in konkreten und nach-konkreten visuellen Texten. Zirndorf.

Wulf, Christoph/Zirfas, Jörg (2005): Bild, Wahrnehmung und Phantasie. Performative Zusammenhänge. In: Wulf, Christoph/Zirfas, Jörg (Hg.): Ikonologie des Performativen. München, S. 7-32.

Wunderlich, Dieter (2008): Spekulationen zum Anfang von Sprache. In: Zeitschrift für Sprachwissenschaft 27.2, S. 229-265.

Zifonun, Gisela/Hoffmann, Ludger/Strecker, Bruno u. a. (1997): Grammatik der deutschen Sprache. 3 Bde. (= Schriften des Instituts für Deutsche Sprache 7.1-7.3). Berlin/New York.

Zweig, Stefan (1997 [1927]): Sternstunden der Menschheit. Vierzehn historische Miniaturen. Frankfurt a.M. 